\title{
Wave propagation in a network of extended Morris-Lecar neurons with electromagnetic induction and its local kinetics
}

\author{
Karthikeyan Rajagopal ${ }^{\mathrm{a},}$, Irene Moroz ${ }^{\mathrm{b}}$, Anitha Karthikeyan ${ }^{\mathrm{a}}$, Prakash Duraisamy ${ }^{\mathrm{c}}$, \\ ${ }^{a}$ Nonlinear Systems and Applications, Faculty of Electrical and Electronics Engineering, Ton \\ Duc Thang University, Ho Chi Minh City, Vietnam. \\ ${ }^{b}$ Mathematical Institute, University of Oxford, Andrew Wiles Building, Oxford, UK. \\ ${ }^{c}$ Center for Nonlinear Dynamics and Control, Mekelle University, Ethiopia. \\ *karthikeyan.rajagopal@tdtu.edu.vn;rkarthiekeyan@gmail.com
}

\begin{abstract}
An extended Morris-Lecar neuron model incorporating electromagnetic flux coupling and external excitation is proposed. The complete dynamical behavior of the new model is investigated as autonomous and non-autonomous forms. Multistability and coexisting attractors are shown for the new neuron model. It is shown that when external excitation is introduced, the complete dynamical behavior of the system changes compared to the nonexcited version. To study the wave propagation pattern, we construct a network of the extended neuron model and study the generation of spiral waves under various conditions of coupling strength, stimuli parameters and system parameters. We have also studied the wave propagation pattern considering the time based chaotic-periodic neurons in the network. Keywords: Morris-Lecar neuron; bifurcation; multistability; spiral waves;
\end{abstract}

\section{Introduction}

Neuron models were developed to understand the activities inside and outside of the neuron to generate signaling process within and among cells [1]. They are used to analyze network dynamics in order to mimic the activity of synaptic connections between neurons. Generally, neurons hold a resting potential of approximately 65 millivolts. In Study of network dynamics on neurons in the last three decades, various models were developed based on the charged particles interacting with the potential of the neurons. In 1955, Hodgkin and Huxley studied the electrical behavior of the giant axon in squid and generate a mathematical model which is the first known mathematical model [5]. Even though the model is efficient, calculating the sodium and potassium conductance makes it practically a challenging one. Following the Hodgkin-Huxley (H-H) approximated model, Fitzhugh-Nagumo in 1952 formulated a simplified two dimensional model to describe the threshold phenomenon in neuron dynamical characteristics [1-4]. Since the dimensionality is low, nonlinear behavior and its analysis can be effectively studied using this model. In 1981 Catherine Morris and Harold Lecar developed a model inspired by the Hodgkin-Huxley model, but using barnacle muscle fibers subjected to stimulation and a different dynamical description [6]. The development of computers enabled various dynamical properties such as bistability. In the M-L model is has three variables: voltage similar to $\mathrm{H}-\mathrm{H}$ model,-fraction of open models, and n-Fraction of open channels. These variables are analogous to the $m$ and $n$ in the Hodgkin-Huxley model, which were ratios 
of these ions inside and outside the cell membrane. Real neurons show a variety of dynamical behaviors: Quiescence, Spiking and Bursting. The M-L model can exhibit various types of spiking. It has hyperbolic tangents and exponents [7].

In [8] Tsumoto et al., presented a general view of the bifurcation mechanism of the 2D M-L model. After that, bifurcation analysis was carried out on the 3D M-L model, Hopf and homoclinic bursting were identified in [9], firing rhythm patterns were generated in [10, 11]. The number of spikes in a bursting periodic orbit have been revealed in [12]. In [13] a 3D M$\mathrm{L}$ model is formulated with memristors and basin of attraction of the asymptotically stable equilibrium point associated with two subcritical Hopf bifurcation points were separated and showed clearly the presence of an unstable limit cycle. In [14], Liu et al., investigated the ML model developed by Prescott et al. [15] with different parameter values and came up with a bistability property. Recently coexisting multiple-stable chaotic firing patterns were revealed in the 3D autonomous and 2D nonautonomous $\mathrm{M}-\mathrm{L}$ neuron models in [16]. By considering various conductance using calcium current, a potassium and a leaky current, a modified M-L model is formulated, dynamical characteristics are analyzed to design an electronic bursting neurons [17]. Literature showing that two coupled M-L models can be synchronized, and synchronization occurs regardless of the initial condition. During two interconnecting neurons if we consider one is periodic spiking and the other is convergent oscillation, then both can be periodic spiking when synchronization occurs [18].

Investigations on if the coupling is neither local nor global, as occurs naturally in certain neural, chemical, and biochemical systems, lay a new platform for researchers. In [19] a spatiotemporal pattern observed in such nonlocally coupled systems. The systems of identical oscillators with symmetrical coupling could self-organize into a state in that some are mutually synchronized while others remained desynchronized. Chimeras are known as complex spatiotemporal patterns that occur during coexistence of both coherent and incoherent groups of coupled systems. The two-dimensional chimeras are identified as spiral waves with a phaserandomized core of desynchronized oscillators surrounded by phase-locked oscillators in the spiral arms [20-22].

In [23] the analytical solution of such a spiral wave chimera was provided, using perturbation methods by calculating the rotation speed and the size of its incoherent core. Dynamical analysis on such spiral waves were carried out [24,25] and some algorithms were developed to suppress spiral waves [26] as well as to avoid the breakup of spiral waves [27]. The interaction with different boundaries causes the motion of spiral waves [28], based on that selection schemes were developed to determine characteristics (shape, rotation frequency) of spiral waves [29]. It has been observed that spiral waves occur not only with an excitable medium [30], sub-excitable medium also generates spiral waves, when noise is forced on the medium [31]. White noise-induced spiral waves and multiple spatial coherence resonances in a neuronal network (M-L) with type I excitability is investigation in [32] and showed that these spiral waves could regulate the wave propagation among neurons. Ali calim et al., [33] investigated the appearance of chimera states in nonlocal networks of type-I Morris-Lecar neurons, coupled via chemical synapses and characterized chimera states in terms of firing frequency and strength of incoherence. Recently a Modified M-L model was studied [34] by blocking the 
target wave with artificial defects and/or partial blocking in ion channels. This revealed that partial channel blocking of some neurons in the network could play a similar role such breaking a target wave with artificial defects. The physical effects of electromagnetic induction while estimating signal encoding and mode selection is discussed in [44] and provided the guidelines to analyze the synaptic plasticity, memristive synapses, and field coupling between neurons and networks.

As can be seen from the literature, most of the M-L neuron model analysis are done in the absence of an electromagnetic induction and external field disturbance. Also, complete chaotic firing phenomenon was not well reported in the earlier literature dealing with M-L model. Secondly the wave propagation phenomenon in a lattice network of M-L neuron was studied in [31] in the absence of electromagnetic induction and hence cannot replicate the biological behavior of the neuron network. Hence this paper investigates the spiral wave generation and wave propagation in a lattice network of a M-L neuron model with electromagnetic induction and external excitation.

\section{Extended Morris-Lecar Neuron model (EMLN)}

The simplest model for the neuron [35] known as the Morris-Lecar (M-L) model with bursting behavior with fast and slow effects, was proposed with three differential equations as

\begin{tabular}{|l|l|}
\hline$C \dot{V}=g_{C a} M_{\infty}\left(V_{C a}-V\right)+g_{K} W\left(V_{K}-V\right)+g_{L}\left(V_{L}-V\right)-I$ \\
$\dot{W}=\tau_{W}\left(W_{\infty}-W\right)$ & \\
$\dot{I}=\varepsilon\left(V_{0}+V\right)$ & \\
\hline
\end{tabular}

where $V$ and $W$ are the fast spiking variables representing the membrane potential and the fraction of open potassium channels respectively while the third state variable, $I$, represents the slow bursting variable. The parameter $\mathrm{C}$ is the capacitance of the membrane while the other system parameters $V_{C a}, V_{K}$, and $V_{L}$ are the steady-state Nernst potentials of calcium ions, potassium ions, and leak channels, respectively. The parameters $g_{C a}$ and $g_{K}$ are the total conductance values for all calcium and potassium while $g_{L}$ is the leak conductance value.

In the M-L model there are three important functions $M_{\infty}, W_{\infty}, \tau_{W}$ representing the stable values of opening probability for all calcium, and potassium channels and time time constant of the potassium activation respectively.

$$
\begin{aligned}
& M_{\infty}=0.5+0.5 \tanh \left(\frac{V-V_{1}}{V_{2}}\right) \\
& W_{\infty}=0.5+0.5 \tanh \left(\frac{V-V_{3}}{V_{4}}\right) \\
& \tau_{W}=\sigma^{-1} \cosh 0.5\left(\frac{V-V_{3}}{V_{4}}\right)
\end{aligned}
$$

It was proposed in [36,41] that inclusion of electromagnetic effects of neuron will show interesting features As further discussed in [37-40], our interest falls on the inclusion of such effects of M-L model (1). The new extended M-L neuron model (EMLN) is 


\begin{tabular}{|l|l|}
\hline$C \dot{V}=g_{C a} M_{\infty}\left(V_{C a}-V\right)+g_{K} W\left(V_{K}-V\right)+g_{L}\left(V_{L}-V\right)-I-k_{0} \rho(\phi) V$ \\
$\dot{W}=\tau_{W}\left(W_{\infty}-W\right)$ & \\
$\dot{I}=\varepsilon\left(V_{0}+V\right)$ & \\
$\dot{\phi}=k_{1} V-k_{2} \phi+\phi_{\text {ext }}$ & \\
\hline
\end{tabular}

where $\rho(\phi)=\alpha+3 \beta \phi^{2}$ is the electromagnetic effect on the neuron and $\phi_{\text {ext }}=E_{1} \cos \left(\omega_{1} t\right)$ is the external excitation. Moving charges will induce a magnetic field, magnetic coupling will trigger and modulate the phase synchronization between two identical neurons connected by electric synapse [43].

The required parameters for simulation are $C=1, V_{C a}=1, V_{L}=-0.5, g_{C a}=1.2, g_{K}=$ $2, g_{L}=0.5, V_{0}=0.2, V_{1}=-0.01, V_{2}=0.15, V_{3}=0.1, V_{4}=0.05, \sigma=3, \varepsilon=0.1, V_{K}=$ $-410, k_{0}=0.1, k_{1}=0.2, k_{2}=1, \alpha=0.1, \beta=0.1, \omega_{1}=1$. In this paper we will discuss the EMLN model with and without external excitations which we call as Case-A $\left(E_{1}=0\right)$ and Case$\mathrm{B}\left(E_{1}=0.1\right)$ respectively. Fig. 1a shows the 2D phase portraits of the EMLN model for Case-A and Case-B. It should be noted that the external excitation if with the fourth state $\phi$, the phase portraits of $\phi-W$ way different in Fig.1a and the time series plots are shown in Fig.1b,1c.
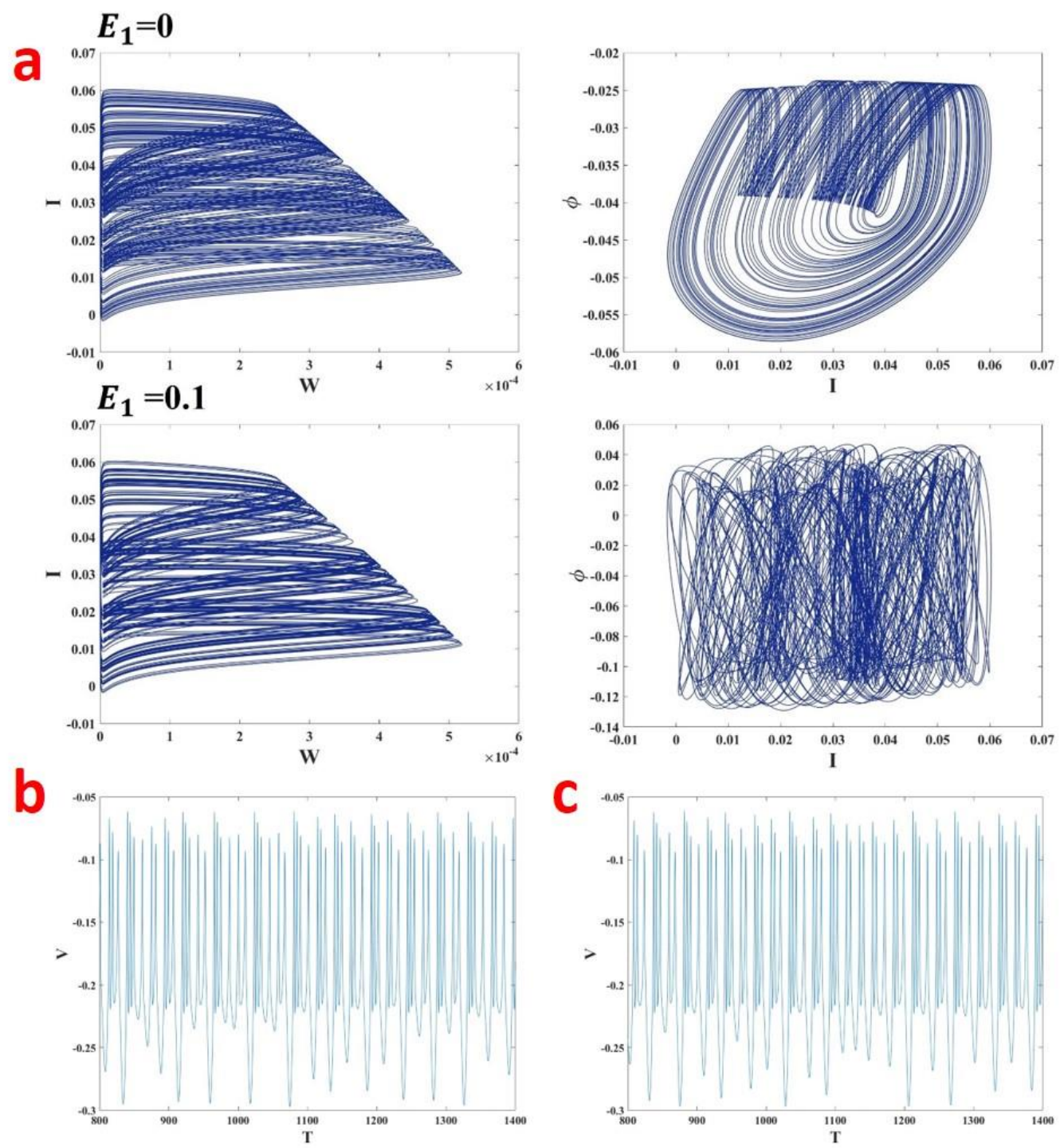
Fig.1a:2D phase portraits of the EMLN with and without excitations. Fig.1b,1C: The time series plots for EMLN without and with external excitations respectively. The required parameters for simulation are $C=1, V_{C a}=1, V_{L}=-0.5, g_{C a}=1.2, g_{K}=2, g_{L}=0.5, V_{0}=$ $0.2, V_{1}=-0.01, V_{2}=0.15, V_{3}=0.1, V_{4}=0.05, \sigma=3, \varepsilon=0.1, V_{K}=-410, k_{0}=0.1, k_{1}=$

$$
0.2, k_{2}=1, \alpha=0.1, \beta=0.1, \omega_{1}=1 \text {. }
$$

\section{Dynamic analysis of EMLN}

The dynamical properties of the EMLN system such as equilibrium points, eigenvalues, Lyapunov spectrum and bifurcation plots are derived and discussed in this section.

\subsection{Equilibrium Points and Stability analysis}

The fixed points of equation (3) are obtained by setting the time derivatives to zero, so that

$$
\begin{aligned}
& V_{e}=-V_{0} \\
& W_{e}=W_{\infty} \\
& \phi_{e}=\frac{-k_{1} V_{0}}{k_{2}} \\
& I_{e}=g c_{a} M_{\infty}\left(V c_{a}-V_{e}\right)+g_{K}\left(V_{K}-V_{e}\right)+g_{L}\left(V_{L}-V_{e}\right)-k_{0} \rho(\phi) V_{e}
\end{aligned}
$$

There is only one fixed point, whose linear stability is found by computing the fourth order Jacobian matrix:

$$
J=\left(\begin{array}{cccc}
F_{V} & F_{W} & F_{I} & F_{\phi} \\
G_{V} & G_{W} & G_{I} & G_{\phi} \\
H_{V} & H_{W} & H_{I} & H_{\phi} \\
L_{V} & L_{W} & L_{I} & L_{\phi}
\end{array}\right)
$$

where, $F_{V}=\frac{\partial F}{\partial V}$, etc. Inspection of the RHS of equations (2) and (3) leads to a simplification of (5):

$$
J_{e}=\left(\begin{array}{cccc}
F_{V} & F_{W} & F_{I} & F_{\phi} \\
G_{V} & G_{W} & 0 & 0 \\
H_{V} & 0 & 0 & 0 \\
L_{V} & 0 & 0 & L_{\phi}
\end{array}\right)
$$

where the Jacobian matrix, evaluated at the fixed point equation (4). determine the roots of the characteristic equation $\operatorname{det}\left(J_{e}-\lambda I_{4}\right)=0$ :

$\lambda^{4}+A_{3} \lambda^{3}+A_{2} \lambda^{2}+A_{1} \lambda+A_{0}=0$ 
Whose coefficients in equation (1.6) are given by

$$
\begin{aligned}
& A_{3}=-\left(F_{V}+G_{W}+L_{\phi}\right) \\
& A_{2}=F_{V}\left(G_{W}+L_{\phi}\right)+G_{W} L_{\phi}-\left(G_{V} F_{W}+F_{I} H_{V}+F_{\phi} L_{V}\right) \\
& A_{1}=L_{\phi}\left(G_{V} F_{W}-F_{V} G_{W}\right)+F_{I} H_{V}\left(G_{W}+L_{\phi}\right)+G_{W} L_{\phi} L_{V} \\
& A_{0}=-F_{I} H_{V} G_{W} L_{\phi}
\end{aligned}
$$

A steady state bifurcation occurs when $A_{0}=0$. Calculations show that

$$
A_{0}=-F_{I} H_{V} G_{W} L_{\phi}
$$

where $F_{I}=-1, H_{V}=\varepsilon, G_{W}=-\tau_{\infty}, L_{\phi}=-k_{2}$. Since the first three terms in this equation cannot vanish, we can only obtain a steady state bifurcation when $k_{2}=0$

We can identify the criteria for a Hopf bifurcation by substituting $\lambda=i \omega$ into equation (7). Equating real and imaginary parts leads to the condition that $\omega^{2}$ must be a common positive root of the quartic $\omega^{4}-A_{2} \omega^{2}+A_{0}=0$ and the quadratic $\omega^{2}=A_{1} / A_{3}$. Eliminating $\omega^{2}$ between these two equations yields the equation for the Hopf bifurcation curve:

\begin{tabular}{|l|l|}
\hline$A_{1}^{2}-A_{1} A_{2} A_{3}+A_{0} A_{3}^{2}=0$ & (10) \\
\hline
\end{tabular}

The parameter $k_{0}$ which appears in equation (4), coupling $V$ to $\phi$, was chosen as one of the key bifurcation parameters. Figure 2 shows the locus of fixed points in the $\left(k_{0}, I\right)$-plane as $k_{0}$ varies. The other expressions in (4) are unchanged. The stability of this fixed point is shown in Figure 3.

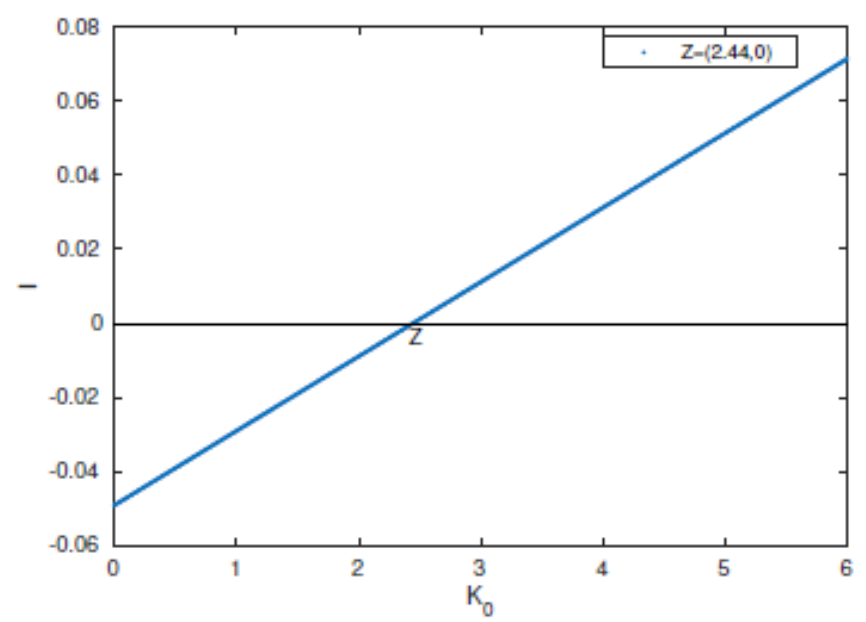

Fig.2: The Locus of the fixed point for the neuron equations as $k_{0}$ varies. The required parameters for simulation are $C=1, V_{C a}=1, V_{L}=-0.5, g_{C a}=1.2, g_{K}=2, g_{L}=0.5, V_{0}=$ $0.2, V_{1}=-0.01, V_{2}=0.15, V_{3}=0.1, V_{4}=0.05, \sigma=3, \varepsilon=0.1, V_{K}=-410, k_{1}=0.2, k_{2}=$ $1, \alpha=0.1, \beta=0.1, \omega_{1}=0$

\subsection{Bifurcation}


From Figure 3, we see there is a Hopf bifurcation at $k_{0} \approx 5.1365$, the remaining two eigen values being real and negative. The Hopf bifurcation is therefore supercritical. Calculations show that the frequency $\omega \approx 0.3268$. This was verified by numerically integrating (3) to obtain

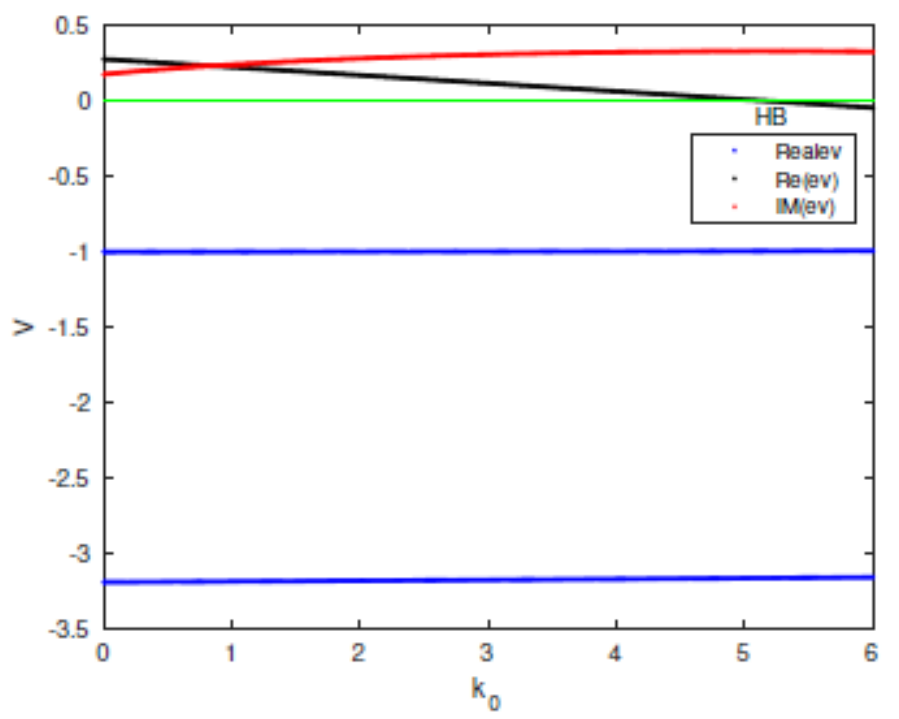

Fig.3: Eigen values for the linear stability of the fixed point (4) as $k_{0}$ varies. The blue curve corresponds to the real eigenvalues, while the black and red curves correspond to the real and imaginary parts of the complex conjugate pair of eigenvalues. The green line indicates where the eigenvalues become pure imaginary, signaling the occurrence of a Hopf bifurcation. The required parameters for simulation are $C=1, V_{C a}=1, V_{L}=-0.5, g_{C a}=1.2, g_{K}=2, g_{L}=$ $0.5, V_{0}=0.2, V_{1}=-0.01, V_{2}=0.15, V_{3}=0.1, V_{4}=0.05, \sigma=3, \varepsilon=0.1, V_{K}=-410, k_{1}=$ $0.2, k_{2}=1, \alpha=0.1, \beta=0.1, \omega_{1}=0$

the bifurcation transition plot of $V_{\max }$ as a function of $k_{0}$, shown in Figure 4a. For small values of $k_{0}$ we find both chaotic and period-3 limit cycles, which each undergo period-halving bifurcations. The lowest branch then disappears when $k_{0} \approx 1.4$, leaving two branches of periodic states, before the lower branch of the two disappears when $k_{0} \approx 2.81$. The upper branch then persists until it disappears in a Hopf bifurcation when $k_{0} \approx 5.1365$ and $V_{\max }=0.2$. We note that when $k_{0}=0.1$ we obtain periodic solutions; in order to obtain chaotic dynamics, we choose $k_{0}=0.05$. We have included the time series plot for different values of $k_{0}$ such that we show chaotic, period- 6 and period- 1 attractors as shown in Fig. $4 b, 4 c, 4 d$ respectively.

If we decrease $k_{0}$ instead, we obtain the bifurcation transition plot of Figure 5. Now we find evidence of more period-halving cascades before the loss of each branch in turn. Moreover there is a noticeable hysteresis, observable if we superimpose the transition plots for $k_{0}$ increasing (red) with that for $k_{0}$ decreasing (see Figure 6). In particular the extra period halving bifurcation windows as $k_{0}$ decreases co-exist with periodic states when $k_{0}$ increases. 


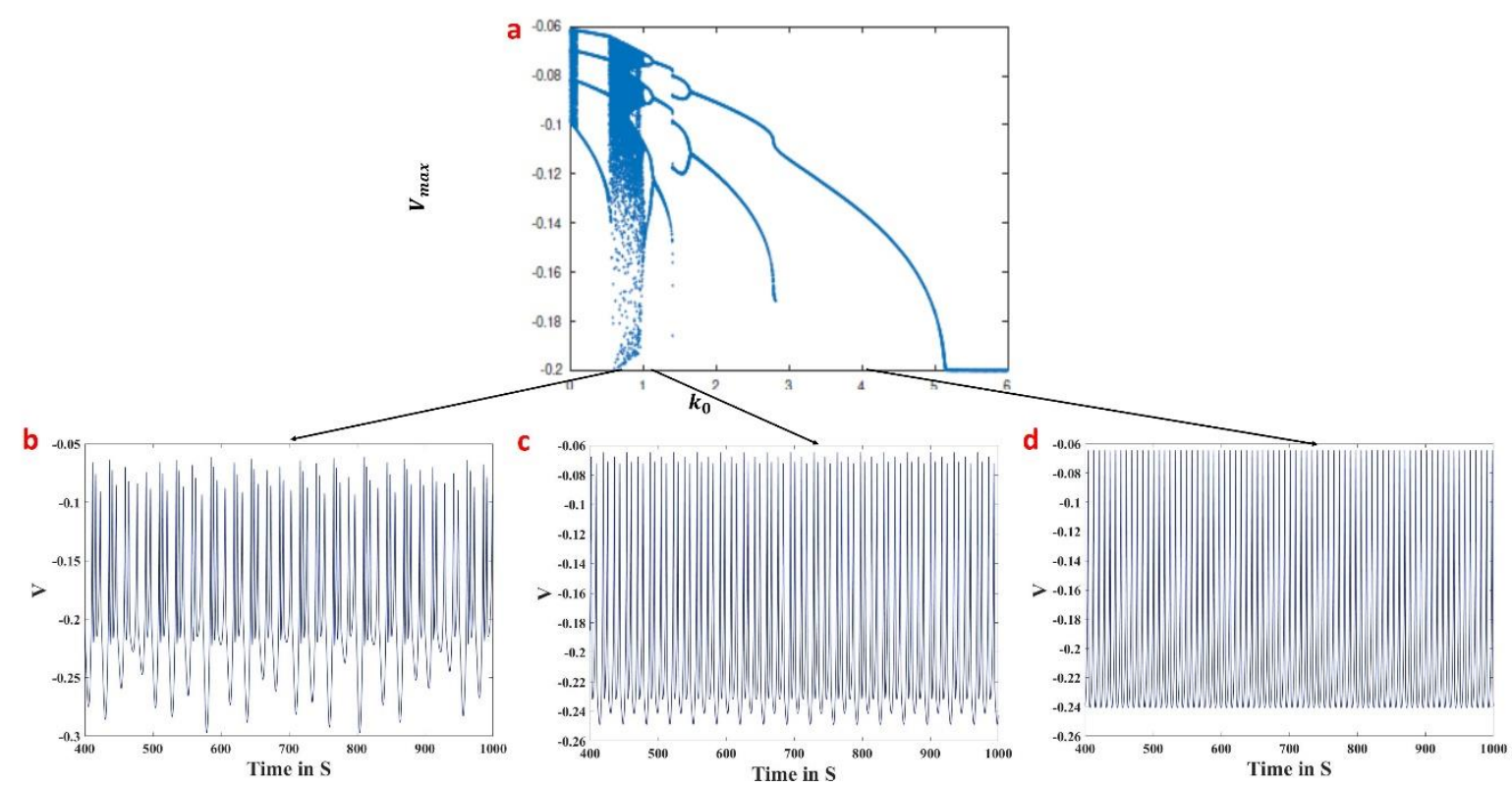

Fig.4a: Bifurcation transition plot of $V_{\max }$ as $k_{0}$ decreases. The Hopf bifurcation at $k_{0} \approx 5.1365$

is clearly visible. Fig. $4 \mathrm{~b}, 4 \mathrm{c}, 4 \mathrm{~d}$ : The time series plots of the system for $k_{0}=0.8,1.1,4$ respectively. The required parameters for simulation are $C=1, V_{C a}=1, V_{L}=-0.5, g_{C a}=$ $1.2, g_{K}=2, g_{L}=0.5, V_{0}=0.2, V_{1}=-0.01, V_{2}=0.15, V_{3}=0.1, V_{4}=0.05, \sigma=3, \varepsilon=$ $0.1, V_{K}=-410, k_{1}=0.2, k_{2}=1, \alpha=0.1, \beta=0.1, \omega_{1}=0$

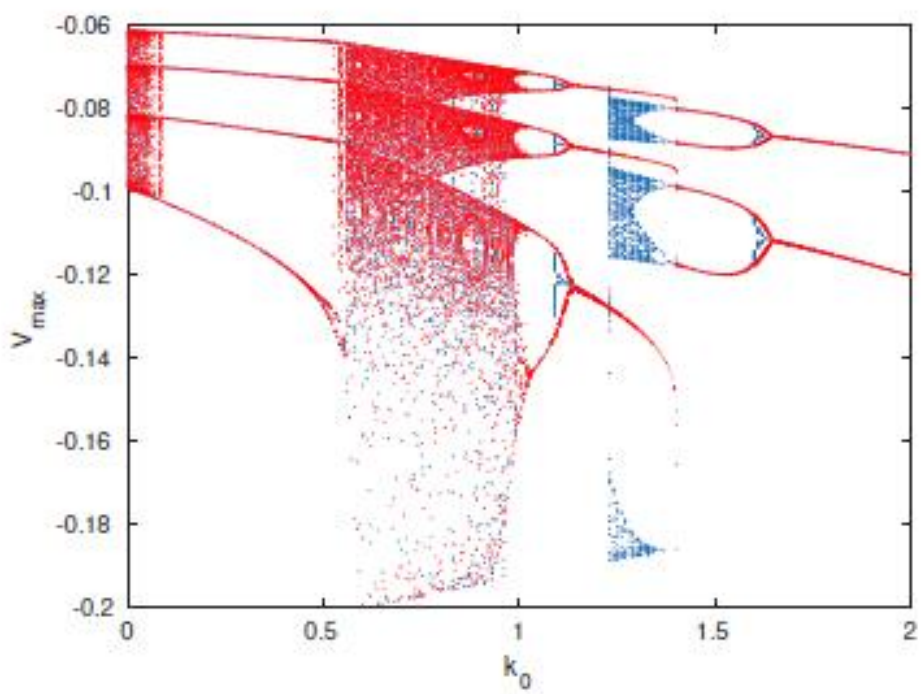

Fig.5: Bifurcation transition plot of $V_{\max }$ as $k_{0}$, increases (red)and decreases (blue).

Hysteresis is clearly visible. The required parameters for simulation are $C=1, V_{C a}=$ $1, V_{L}=-0.5, g_{C a}=1.2, g_{K}=2, g_{L}=0.5, V_{0}=0.2, V_{1}=-0.01, V_{2}=0.15, V_{3}=$ $0.1, V_{4}=0.05, \sigma=3, \varepsilon=0.1, V_{K}=-410, k_{1}=0.2, k_{2}=1, \alpha=0.1, \beta=0.1, \omega_{1}=0$

\subsection{Multistability and coexisting attractors:}


It should be noted that the bifurcation plots shown in Fig.4 and Fig.5 are without external excitation. To better understand the multistability nature of the EMLN system we can also vary the parameter $V_{k}$, which appears in equation (3). For the first case we consider that the system is autonomous and doesn't have external excitation. Using forward and backward continuation in which either the parameter is either increased or decreased with reinitializing of initial conditions to end values of state trajectories we plot the local maxima of $z$. The outcome is shown in Figure 6a where red dots show backward continuation and blue dots show forward continuation. We have a series of period halving cascades, separated by regions of chaotic dynamics. We commence with four branches, which reduce by one after each region of chaos, followed by the period-halving bifurcations, until just one branch of periodic solutions remains. To be more specific the EMLN shows chaotic attractors for $-535 \leq V_{K} \leq-429$, $-416.5 \leq V_{K} \leq-388$ and $-375 \leq V_{K} \leq-358$. We could also see coexisting chaotic and period -4 attractors for $-535 \leq V_{K} \leq-533.7$ and coexisting period-4 and period-3 limit cycles for $-538 \leq V_{K} \leq-547$. The Maximum two Lyapunov exponents (LEs) are calculated using Wolf's method [42], and plotted for a finite time of 20000s as shown in Fig.6b and 6c.
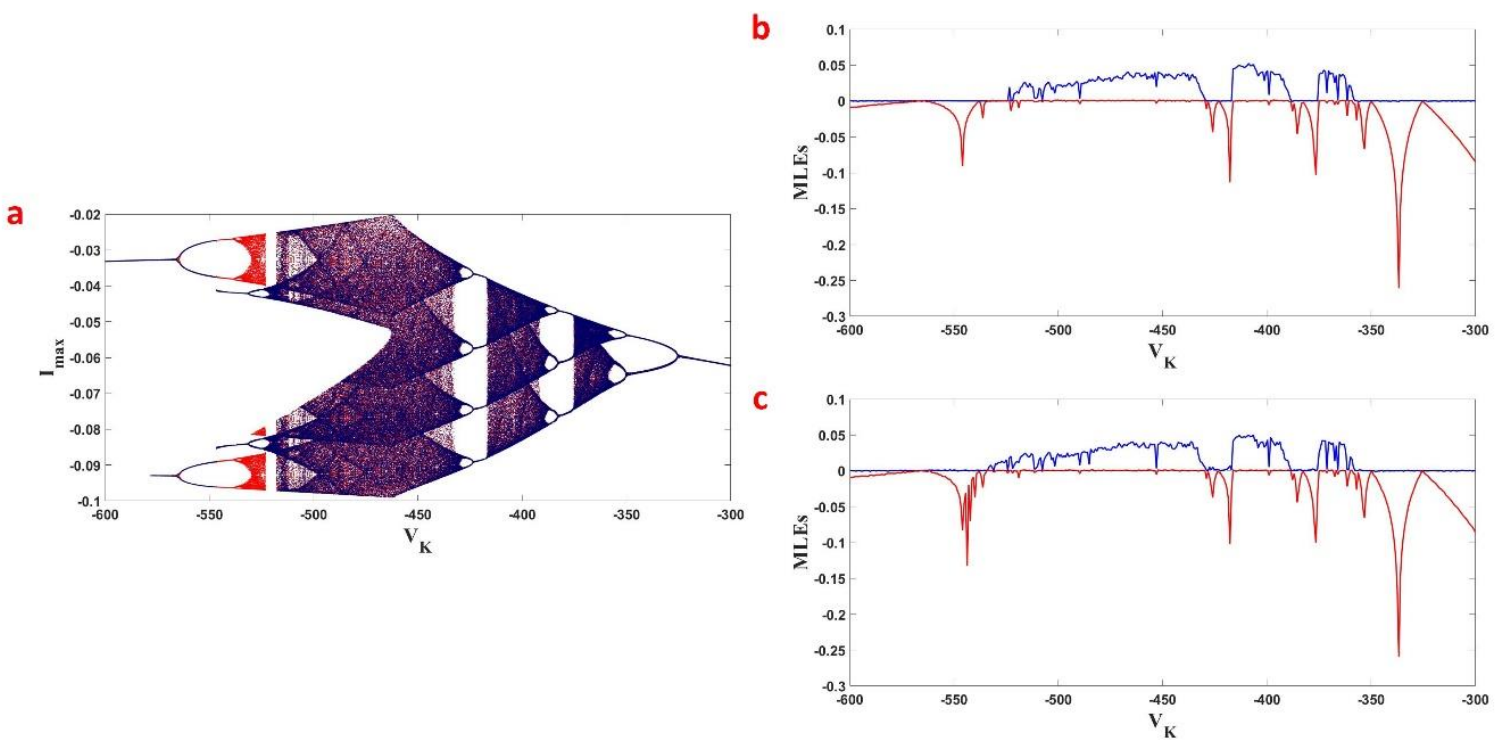

Fig.6:a) Bifurcation of the autonomous EMLN system (case-A) with $V_{K}$ with red color showing backward and blue color showing forward continuation; b) Maximum two LEs for forward continuation; c) Maximum two LEs for backward continuation. The required parameters for simulation are $C=1, V_{C a}=1, V_{L}=-0.5, g_{C a}=1.2, g_{K}=2, g_{L}=0.5, V_{0}=$ $0.2, V_{1}=-0.01, V_{2}=0.15, V_{3}=0.1, V_{4}=0.05, \sigma=3, \varepsilon=0.1, k_{0}=0.1, k_{1}=0.2, k_{2}=$ $1, \alpha=0.1, \beta=0.1, \omega_{1}=0$

In our second discussion we investigate the bifurcation and multistability in a forced EMLN model. For this we consider external excitation as $\phi_{\text {ext }}=E_{1} \cos \left(\omega_{1} t\right)$ and use forward and backward continuation to plot the local maxima of $z$. While using backward continuation (blue dots) we could see that the chaotic oscillations exists for $-600 \leq V_{K} \leq-450$ whereas when using backward continuation, we could see chaos existence for $-500 \leq V_{K} \leq-330$ with series of period halving cascades, separated by regions of chaotic dynamics. To better 
understand the dynamical behavior of the excited EMLN system we have now plotted the phase plots for $V_{K}=-400$ for two different initial conditions as shown in Fig. $8 \mathrm{c}$ and $8 \mathrm{~d}$. We see that the EMLN shows chaotic attractors when using the initial condition $[0,0,0,0.5]$ out shows quasi-periodic oscillations for $[-1,-1,-1,-1]$. To verify this we have plotted the corresponding MLEs for forward and backward continuation which are shown in Fig.7b and Fig.7c respectively.
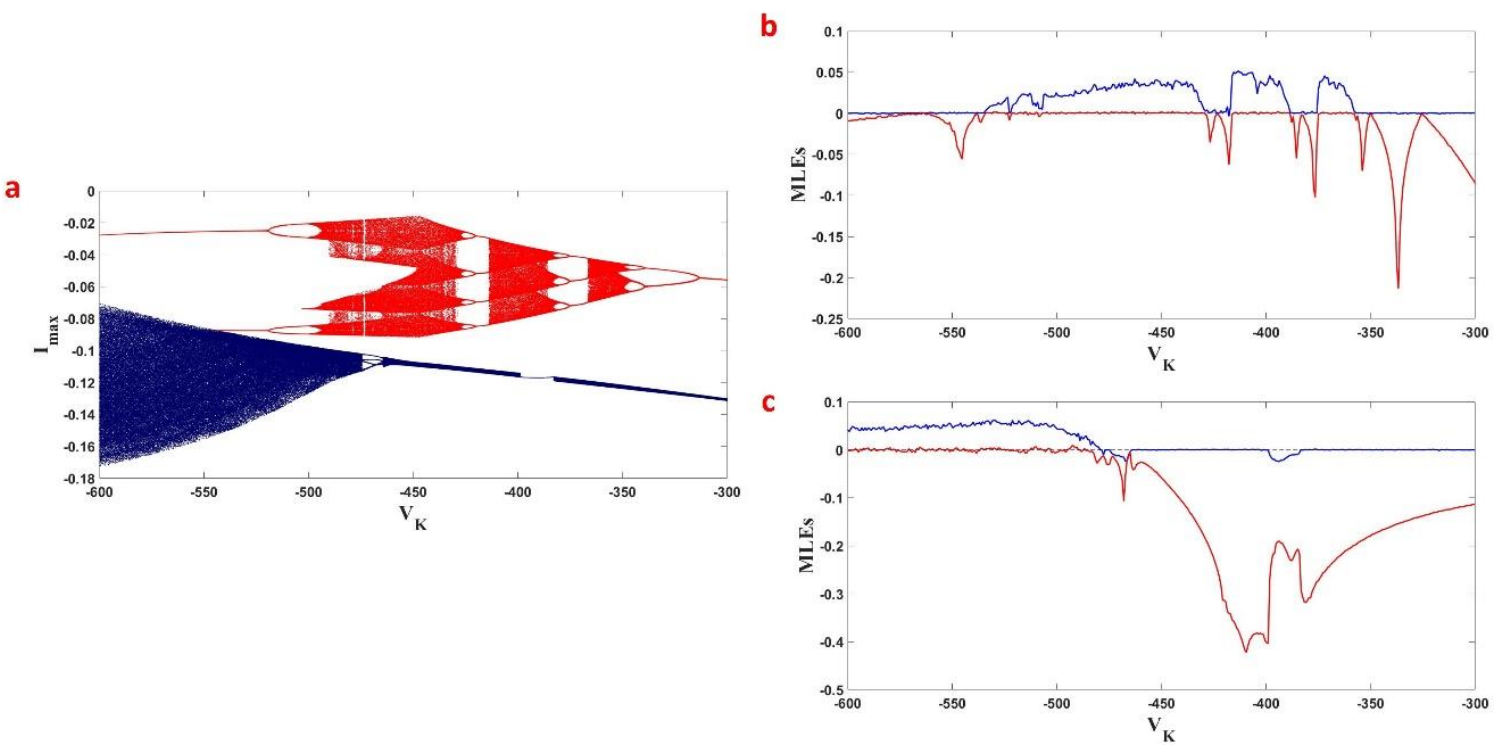

Fig.7:a) Bifurcation of the excited EMLN system (case-b) with $V_{K}$ with red color showing backward and blue color showing forward continuation; b) Maximum two LEs for forward continuation; c) Maximum two LEs for backward continuation. The required parameters for

$$
\begin{gathered}
\text { simulation are } C=1, V_{C a}=1, V_{L}=-0.5, g_{C a}=1.2, g_{K}=2, g_{L}=0.5, V_{0}=0.2, V_{1}= \\
-0.01, V_{2}=0.15, V_{3}=0.1, V_{4}=0.05, \sigma=3, \varepsilon=0.1, k_{0}=0.1, k_{1}=0.2, k_{2}=1, \alpha= \\
0.1, \beta=0.1, E_{1}=1, \omega_{1}=1
\end{gathered}
$$

The coexisting attractors for both case-A and case-B are shown in Fig.8a and Fig.8b respectively. We see that the autonomous EMLN system shows coexisting period-2 oscillation with a chaotic attractor, and in the excited EMLN system, a period-4 limit cycle coexists with chaotic attractor. 

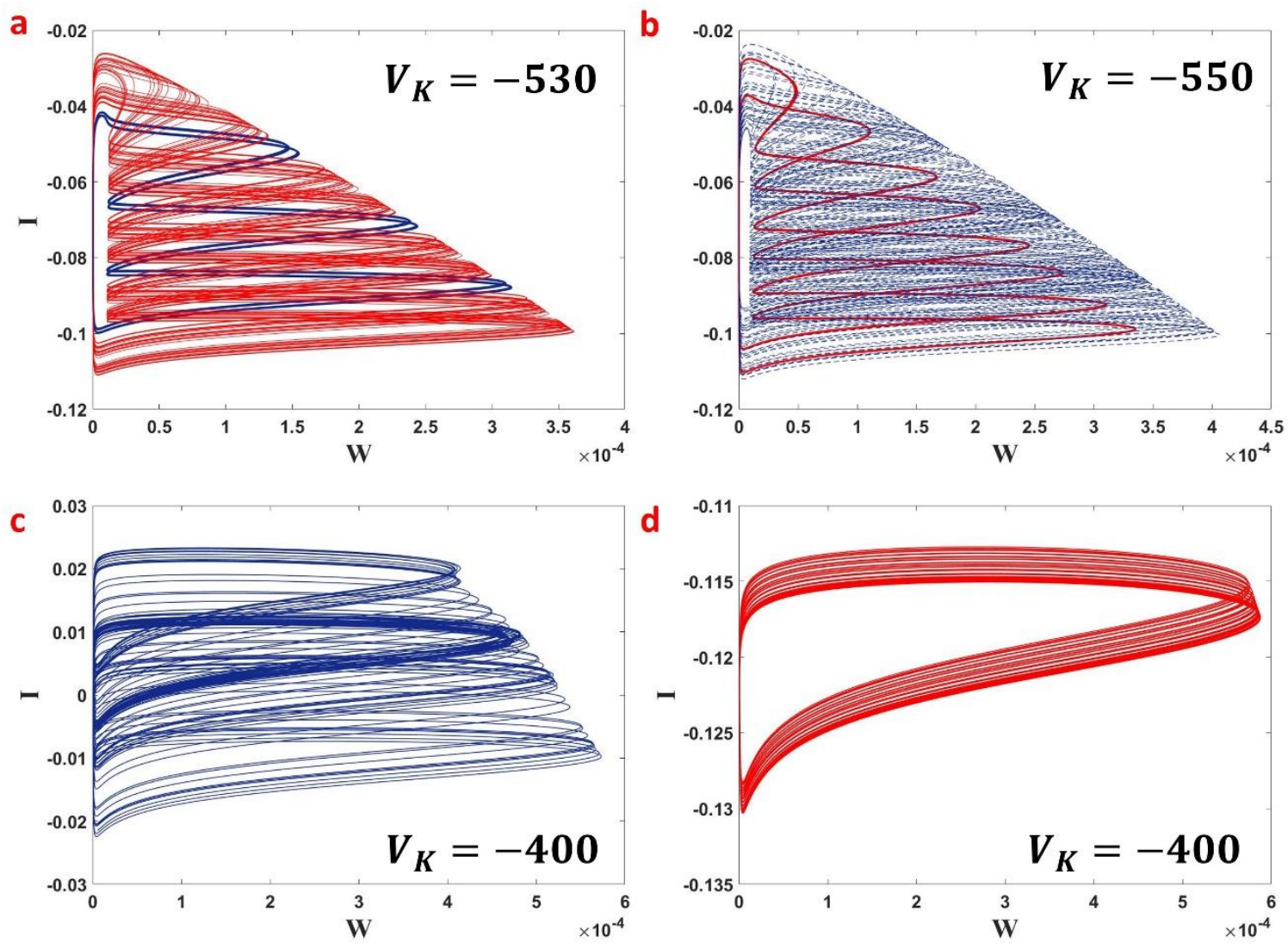

Fig.8: a) Coexisting limit cycle and chaotic attractor for $V_{K}=-530$ in an autonomous

ELMN system for the initial conditions $[-0.13,0,-0.07,-0.07]$ for blue plot and $[-0.21,0,-0.1,-0.09]$ for red plot; b) Coexisting limit cycle and chaotic attractor for $V_{K}=$ -550 in an nonautonomous ELMN system for the initial conditions [0,0,0,0.5] for red plot and $[-0.28,0,-0.04,-0.09]$ for blue plot. Fig.8(c,d): The $2 \mathrm{D}$ phase plots of a nonautonomous ELMN for $V_{K}=-400$ with the initial conditions $[0,0,0,0.5]$ shown in Fig.8c and $[-1 .-1,-1,-1]$ shown in Fig.8d.The required parameters for simulation are $C=1, V_{C a}=$ $1, V_{L}=-0.5, g_{C a}=1.2, g_{K}=2, g_{L}=0.5, V_{0}=0.2, V_{1}=-0.01, V_{2}=0.15, V_{3}=0.1, V_{4}=$ $0.05, \sigma=3, \varepsilon=0.1, k_{0}=0.1, k_{1}=0.2, k_{2}=1, \alpha=0.1, \beta=0.1, \omega_{1}=0$

\section{Spiral waves in EMLN model}

To investigate the phenomenon of wave propagation and the formation of spiral waves in the EMLN system, we construct a network of $110 \times 110$. The network constructed is a twodimensional lattice structure with interactions only of neighboring neurons. The coupling strength between the neighboring neurons is $D$. One sinusoidal stimulation $(A \cos (\omega t))$ is considered which activates the start time of 30 and end time of 800. This stimulus is applied to the center of the medium and propagates to the left and right boundaries. For the numerical integration of the network we use the Runge-Kutta $4^{\text {th }}$ order considering that there is no-flux boundary condition. The initial states of the variables are $(x, y, z, \varphi)=(0,0,0,0.5)$ and the calculating period is about 5000-time units.

The spatiotemporal pattern of the membrane potential is observed, and snapshots of the resulting patterns are displayed in $\mathrm{t}=5000$-time units. We have considered two types of EMLN 
network. In the first type we assume that all the neurons are in the same state (chaotic or periodic) depending on the value of the parameter $V_{K}$ for the entire simulation time. In the second type, we consider that for the first 2000-time units, the EMLN is in chaotic state with $V_{K}=-410$ and for the remaining 5000-time units the EMLN is either in a chaotic or periodic state, depending on the choice of $V_{K}$. Such an investigation will show us the behavior of the neuron network and the wave propagation phenomenon of neuronal systems which changes its behavior with time. In each type we consider the autonomous EMLN (case-A) and nonautonomous EMLN (case-B). Various investigations are made to study the effects of the coupling strength $D$, the stimuli amplitude $A$ and stimuli frequency $\omega$. Similarly, the effects of the parameter $V_{K}$ on the wave propagation in EMLN system is also studied.

The EMLN network of $110 \times 110$ for type- 1 can be defined as,

$$
\begin{aligned}
& C \dot{V}_{i j}=g_{C a} M_{\infty}\left(V_{C a}-V_{i j}\right)+g_{K} W\left(V_{K}-V_{i j}\right)+g_{L}\left(V_{L}-V_{i j}\right)-I_{i j}-k_{0} \rho\left(\phi_{i j}\right) V_{i j} \\
& +D\left(V_{i+1 j}+V_{i-1 j}+V_{i j+1}+V_{i j-1}-4 V_{i j}\right)+\psi(t) \zeta_{i \theta_{1}} \zeta_{j \theta_{2}} \\
& \dot{W}_{i j}=\tau_{W}\left(W_{\infty}-W_{i j}\right) \\
& \dot{I}_{i j}=\varepsilon\left(V_{0}+V_{i j}\right) \\
& \dot{\phi}_{i j}=k_{1} V_{i j}-k_{2} \phi_{i j}+\phi_{e x t}
\end{aligned}
$$

where $\psi(t)=A \cos (\omega t)$ is the induced force and $\zeta_{i \theta_{1}}=1, \zeta_{j \theta_{2}}=1$ exists for $i=\theta_{1}, j=\theta_{2}$ respectively.

For the EMLN network of type- 2 we consider a $110 \times 110$ network as,

$$
\begin{aligned}
C \dot{V}_{i j}= & g_{C a} M_{\infty}\left(V_{C a}-V_{i j}\right)+g_{K} W\left(V_{K}(c)-V_{i j}\right)+g_{L}\left(V_{L}-V_{i j}\right)-I_{i j}-k_{0} \rho\left(\phi_{i j}\right) V_{i j} \\
& +D\left(V_{i+1 j}+V_{i-1 j}+V_{i j+1}+V_{i j-1}-4 V_{i j}\right)+\psi(t) \zeta_{i \theta_{1}} \zeta_{j \theta_{2}} \\
\dot{W}_{i j}= & \tau_{W}\left(W_{\infty}-W_{i j}\right) \\
\dot{I}_{i j}= & \varepsilon\left(V_{0}+V_{i j}\right) \\
\dot{\phi}_{i j}= & k_{1} V_{i j}-k_{2} \phi_{i j}+\phi_{e x t}
\end{aligned}
$$

where

$$
\left\{\begin{array}{l}
V_{K}(c)=-410 \text { for } 0 \leq t<2000 \\
V_{K}(c)=V_{K} \text { for } 2000 \leq t<5000
\end{array}\right.
$$

with $V_{K}$ taking the values of $[-200,-300,-400,-500,-600,-700,-800,-900]$. The stimuli is $\psi(t)=A \cos (\omega t)$ and $\psi(t)$ is the momentary force which only exists if $\zeta_{i \theta_{1}}=1, \zeta_{j \theta_{2}}=1$ exists for $i=\theta_{1}, j=\theta_{2}$ respectively. The $\psi(t)$ is imposed on the central part of the network by setting $\theta_{1}=\theta_{2}=50$. 
Further the discussion is divided in to Case-A: when $\phi_{e x t}=0$, Case-B: when the excitation is periodic $\left(\phi_{\text {ext }}=E_{1} \cos \left(\omega_{1} t\right)\right.$ where $E$ is the intensity of the external excitation and $\omega$ is the frequency of excitation). Also, we have considered no-flux boundary condition defined as

$$
\frac{\partial V}{\partial \beta}=\frac{\partial W}{\partial \beta}=\frac{\partial I}{\partial \beta}=\frac{\partial \phi}{\partial \beta}=0
$$

where $\beta$ is the outward normal vector to the $2 \mathrm{D}$ plane.

\subsection{Wave propagation in a type-1 network with no excitation:}

Our first discussion will be on the type-1 EMLN network as defined in (11) without external excitation (case-A). The investigation is subdivided in to four section where in we discuss the effect of the amplitude $A$ of the external stimuli with the frequency and coupling coefficient at $D=1, \omega=0.1$. In the second discussion we investigate the effects of the stimuli frequency ( $\omega$ ) by keeping $D=1, A=0.1$. The third discussion will be on the effect of coupling coefficient $D$ with $\omega=0.1, A=0.1$. In all the above three discussions the parameter $V_{K}=$ -410 and the other system parameters are kept as in Fig.1. Final discussion will be on the effect of parameter $V_{K}$ on the wave propagation pattern.

\subsubsection{Effect of the stimuli amplitude $A$}

The stimuli amplitude is varied as $A=[0.001,0.01,0.1,0.2,0.25,0.3,0.35]$ and the snapshots of the spatiotemporal behavior are captured as shown in Fig.9. For $A=0.001$ we could see the spiral wave formation which are then deformed when the amplitude is increased to $A=0.01$. Further increasing the amplitude to $A=0.1$ again induces spiral waves in the network which are then supported by further amplitude increases to $A=0.2$ and $A=0.25$. When the amplitude is increased to $A=0.3$ the spiral waves are affected by the amplitude of the external stimuli and hence starts merging which can see being deformed when $A=0.35$. This shows that when the external stimuli amplitude is increased, the spiral waves are affected by the stimuli and get dissipated. 

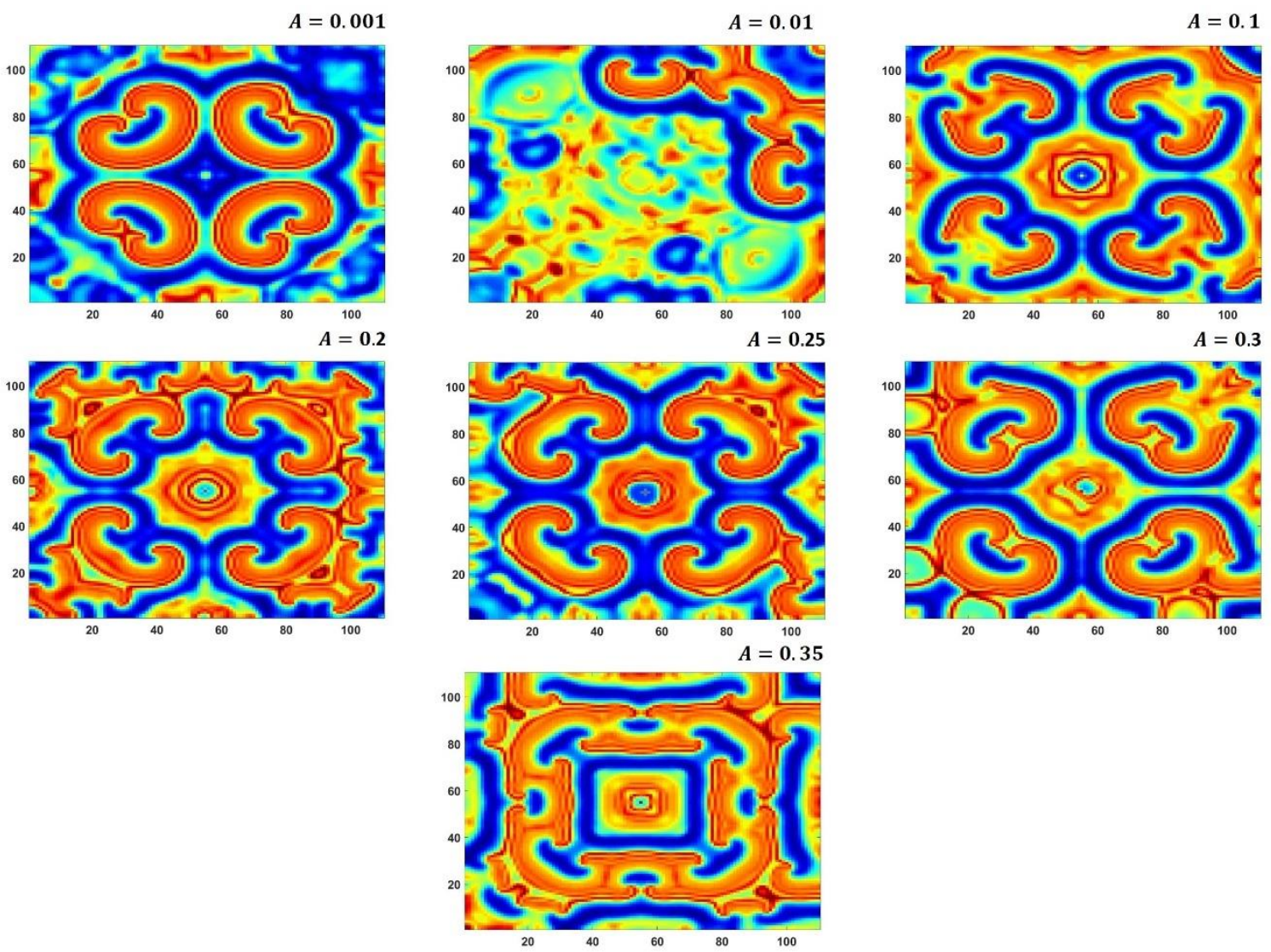

Fig.9: Spatiotemporal snap shots of the membrane potential for Type-1 autonomous (Case-A) EMLN network for various values of the stimuli amplitude $A$ with $\omega=0.1$ and $D=1$. The required parameters for simulation are $C=1, V_{C a}=1, V_{L}=-0.5, g_{C a}=1.2, g_{K}=2, g_{L}=$

$$
\begin{gathered}
0.5, V_{0}=0.2, V_{1}=-0.01, V_{2}=0.15, V_{3}=0.1, V_{4}=0.05, \sigma=3, \varepsilon=0.1, k_{0}=0.1, k_{1}= \\
0.2, k_{2}=1, \alpha=0.1, \beta=0.1, E_{1}=1, \omega_{1}=0
\end{gathered}
$$

\subsubsection{Effect of the stimuli amplitude $\omega$}

Understanding the effect of stimuli amplitude on the wave propagation in a type-1 EMLN network, our interest now focusses on the stimuli frequency ( $\omega$ ). Taking $A=0.1$ and $D=1$, we show the snapshots of the EMLN network at 5000-time units for various values of $\omega=$ $[0.0001,0.001,0.01,0.1,0.3,0.5,0.8,1,2,5]$ in Fig. 10 . When the stimuli frequency is low at 0.0001 and 0.001 we observe that the spiral waves originate at the corners of the network when the wave propagates from the center of the network. The spiral seeds soon form a more definable spiral wave when $\omega=0.01$ and starts degrading for $\omega=0.1$, and thus loses the spiral seeds as the frequency increases to $\omega=0.2$. Further increasing the frequency we could again see the regeneration of the spiral waves, this time much stronger with multiple cascading spiral waves seen for $\omega=1$ and the cascaded spiral waves forming their one resonating pools containing many spiral seeds for $\omega=2$, Further increasing the frequency to $\omega=5$, the spiral pool is washed away and thus there is no more regeneration of spiral waves. 

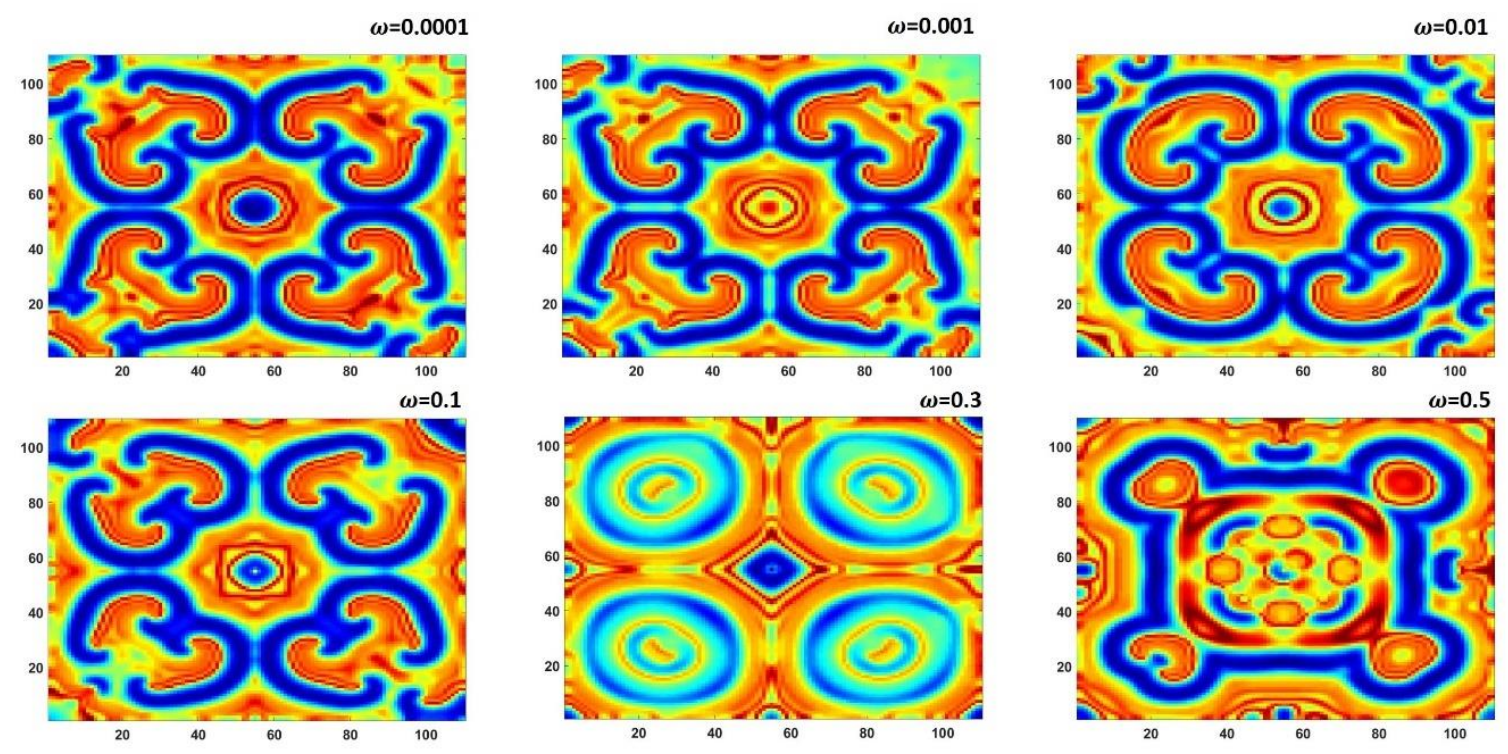

$\omega=0.8$

$\omega=1$
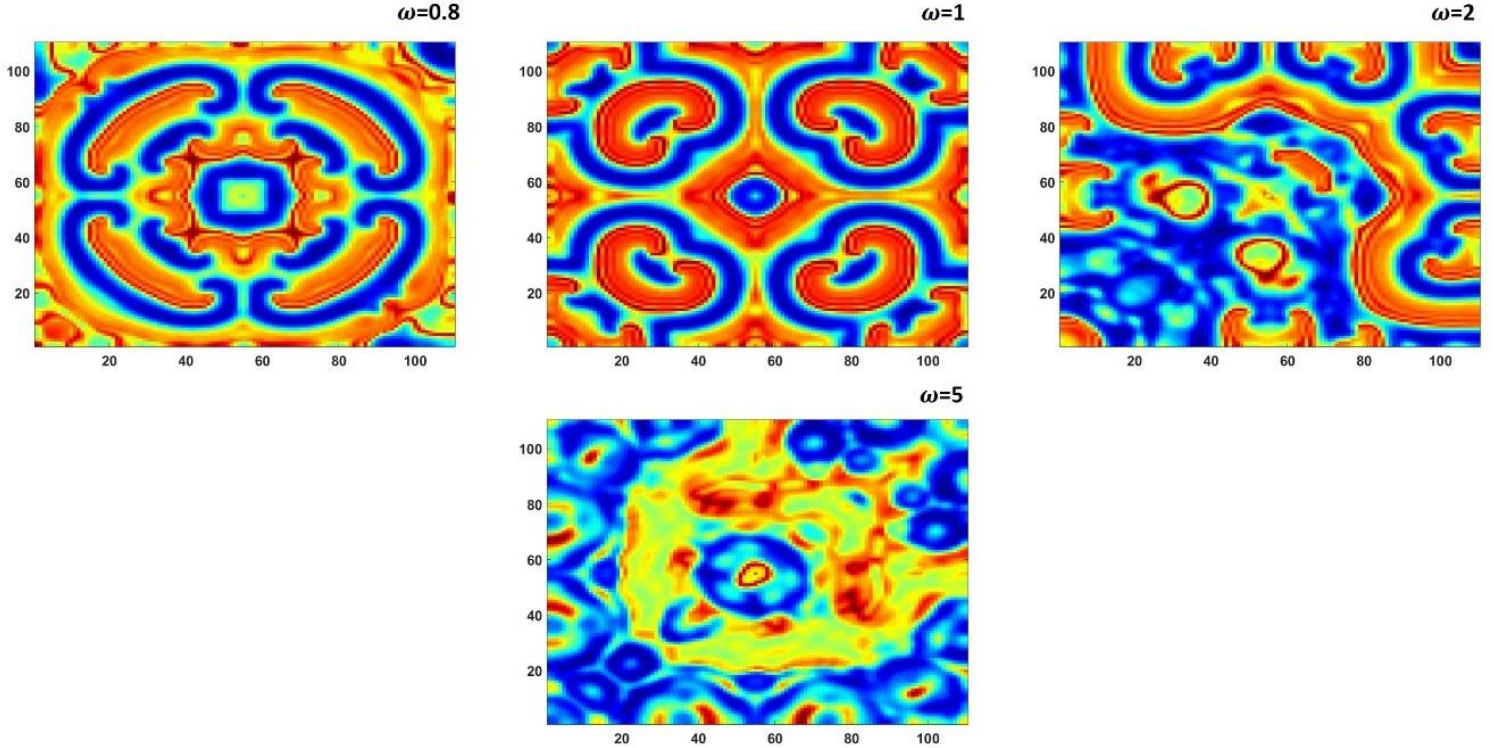

Fig.10: Spatiotemporal snap shots of the membrane potential for Type-1 autonomous (CaseA) EMLN network for various values of the stimuli frequency $\omega$ with $A=0.1$ and $D=1$.

The required parameters for simulation are $C=1, V_{C a}=1, V_{L}=-0.5, g_{C a}=1.2, g_{K}=$ $2, g_{L}=0.5, V_{0}=0.2, V_{1}=-0.01, V_{2}=0.15, V_{3}=0.1, V_{4}=0.05, \sigma=3, \varepsilon=0.1, k_{0}=$ $0.1, k_{1}=0.2, k_{2}=1, \alpha=0.1, \beta=0.1, E_{1}=1, \omega_{1}=0$

\subsubsection{Effect of the stimuli amplitude D}

The coupling strength between the interacting neurons is the most essential component to be discussed when we want to investigate the wave propagation. Hence, we fix the amplitude and frequency of the stimuli at $A=0.1 ; \omega=0.1$ and study the wave propagation pattern for different coupling strengths $D=[10 \%, 30 \%, 50 \%, 70 \%, 80 \% 100 \%]$. Fig. 11 shows the spatiotemporal behavior of the type-1 EMLN network for various coupling strengths as the coupling strength increases, the spiral seeds are formed by the interacting neurons and thus finally ends up forming spiral waves for $100 \%$ coupling. 

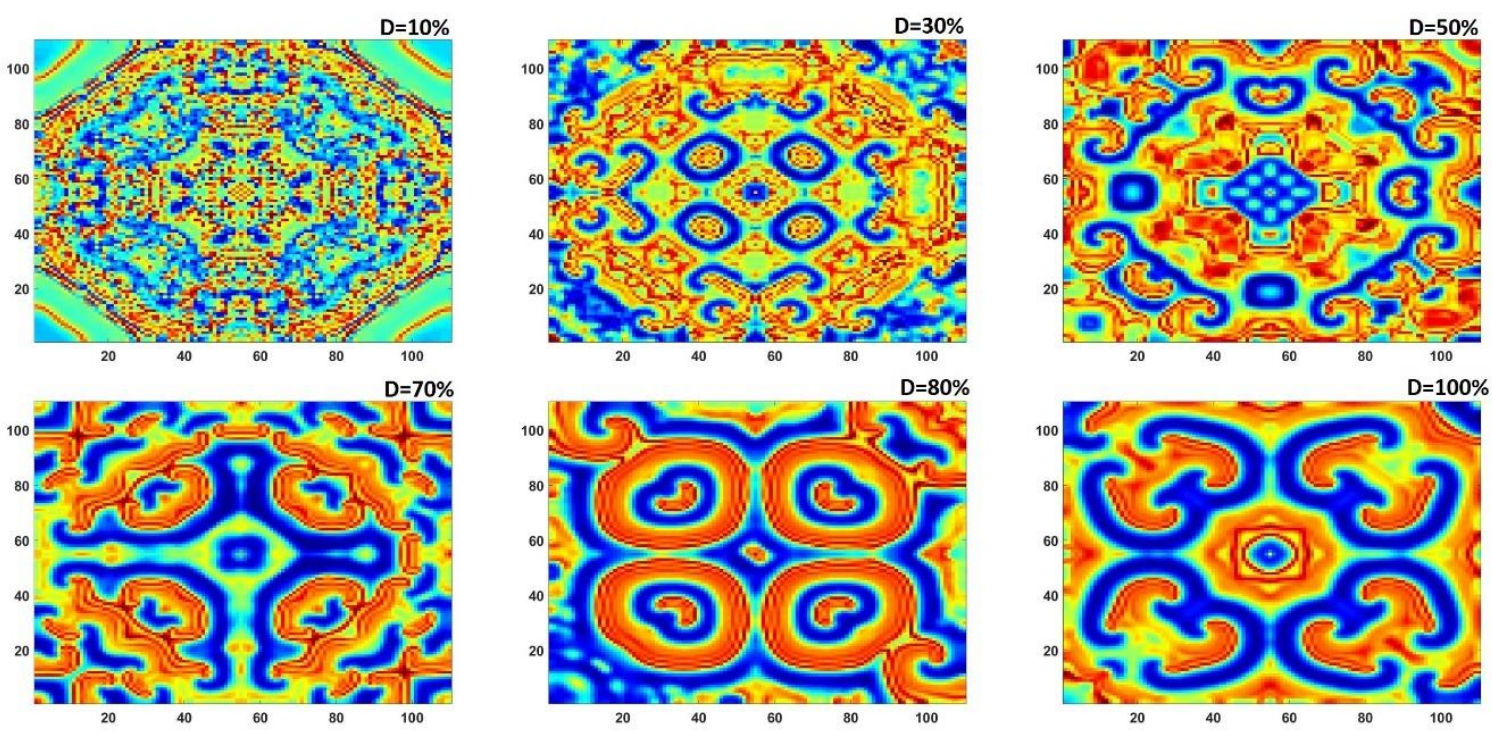

Fig.11: Spatiotemporal snap shots of the membrane potential for Type-1 autonomous (Case-

A) EMLN network for various values of the $D$ with $A=0.1$ and $\omega=0.1$. The required parameters for simulation are $C=1, V_{C a}=1, V_{L}=-0.5, g_{C a}=1.2, g_{K}=2, g_{L}=0.5, V_{0}=$ $0.2, V_{1}=-0.01, V_{2}=0.15, V_{3}=0.1, V_{4}=0.05, \sigma=3, \varepsilon=0.1, k_{0}=0.1, k_{1}=0.2, k_{2}=$ $1, \alpha=0.1, \beta=0.1, E_{1}=1, \omega_{1}=0$

\subsubsection{Effect of the Nernst potential $V_{K}$}

To completely understand the wave propagation in the EMLN network it is important to study the parameter effects. For this we consider the Nernst potential of potassium ion $\left(V_{K}\right)$ and investigate the wave propagation pattern. As we know from Fig.6a, the autonomous EMLN system shows cascade of chaotic regions for $-535 \leq V_{K} \leq-350$ and hence we start from $V_{K}=-300$ which as seen from Fig.12 is not showing any evidence of spiral waves. Further decreasing the potential to -350 shows the accumulation of the waves in the four corners of the network but still no spiral seeds are seen. At $V_{K}=-400$ we see the formation of multiple spiral seeds which are then grown to a complete spiral wave at $V_{K}=-450$. At $V_{K}=-500$ the spiral waves are dissipated in the neuron network and when further decreased to $V_{K}=-550$ we see the rebirth of spiral waves, this time with a much larger resonant amplitude 

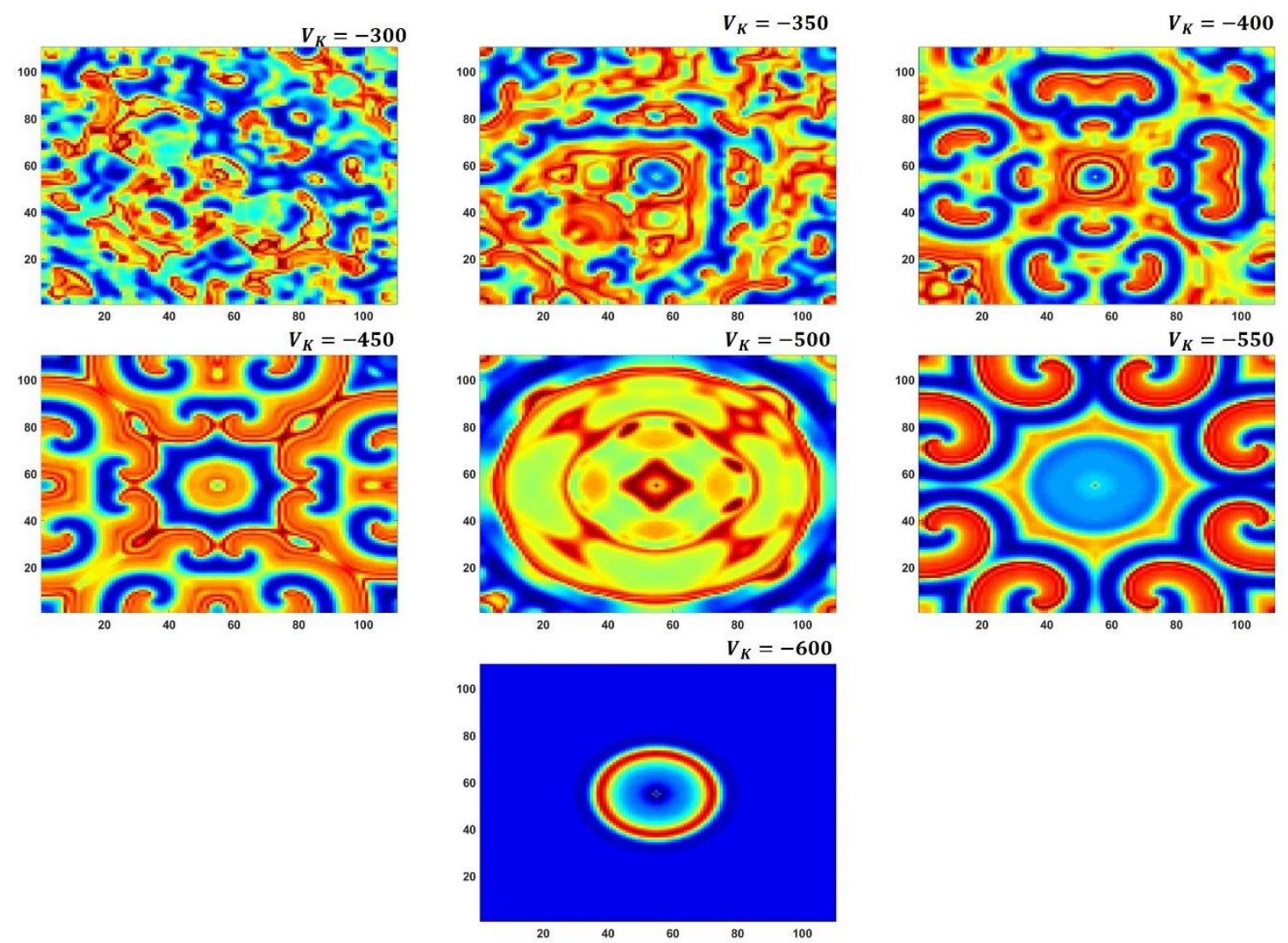

Fig.12: Spatiotemporal snap shots of the membrane potential for Type-1 autonomous (CaseA) EMLN network for various values of the Nernst potential $V_{K}$ with $A=0.1, \omega=0.1$ and $D=1$. The required parameters for simulation are $C=1, V_{C a}=1, V_{L}=-0.5, g_{C a}=$ $1.2, g_{K}=2, g_{L}=0.5, V_{0}=0.2, V_{1}=-0.01, V_{2}=0.15, V_{3}=0.1, V_{4}=0.05, \sigma=3, \varepsilon=$ $0.1, k_{0}=0.1, k_{1}=0.2, k_{2}=1, \alpha=0.1, \beta=0.1, E_{1}=1, \omega_{1}=0$

\subsection{Wave propagation in a type-1 network with external excitation:}

Our next discussion will be on the type-1 externally excited EMLN network (case-B). Similar to the discussions in section 4.1, we investigate the effect of the stimuli amplitude $A$, stimuli frequency $\omega$ and the coupling strength $D$ of the external stimuli, considering the frequency and coupling coefficient and keeping the parameter $V_{K}=-410$ and the other system parameters are kept as in Fig.1. Finally, we investigate the effects of parameter $V_{K}$ on the wave propagation.

\subsubsection{Effect of the stimuli amplitude A}

To analyze the impact of stimuli amplitude $A$ on the wave propagation in the type-1 EMLN network, we consider $100 \%$ coupling and stimuli frequency at $\omega=0.1$. The amplitude of the external excitation is taken as $E=0.1$ and frequency $\varphi=1$ with the parameter $V_{K}=-410$. The various values of stimuli amplitude $A$ are taken as $[0.001,0.01,0.1,0.5,1,1.5,2,2.2]$. Fig.13 shows the spatiotemporal snapshots of the wave propagation in the network at 5000-time units. At the amplitude value of $A=0.001$, we see the emergence of multiple spiral seeds which are 
then further increased when $A=0.01$. Further increase in the amplitude to $A=0.1$ The spiral wave seeds get organized at the corners of the network and start resonating with the stimuli. For the amplitude $A=0.5$ the spiral waves starts to multiply along with the stimuli as the entire network propagates the wave, and shows that the chaotic states of the system takes the stimuli wave as the excitation to their chaotic states. Further increase in amplitude starts to influence the propagation of the spiral waves and soon starts to degrade the wave propagation and there by causing the death of spiral waves.

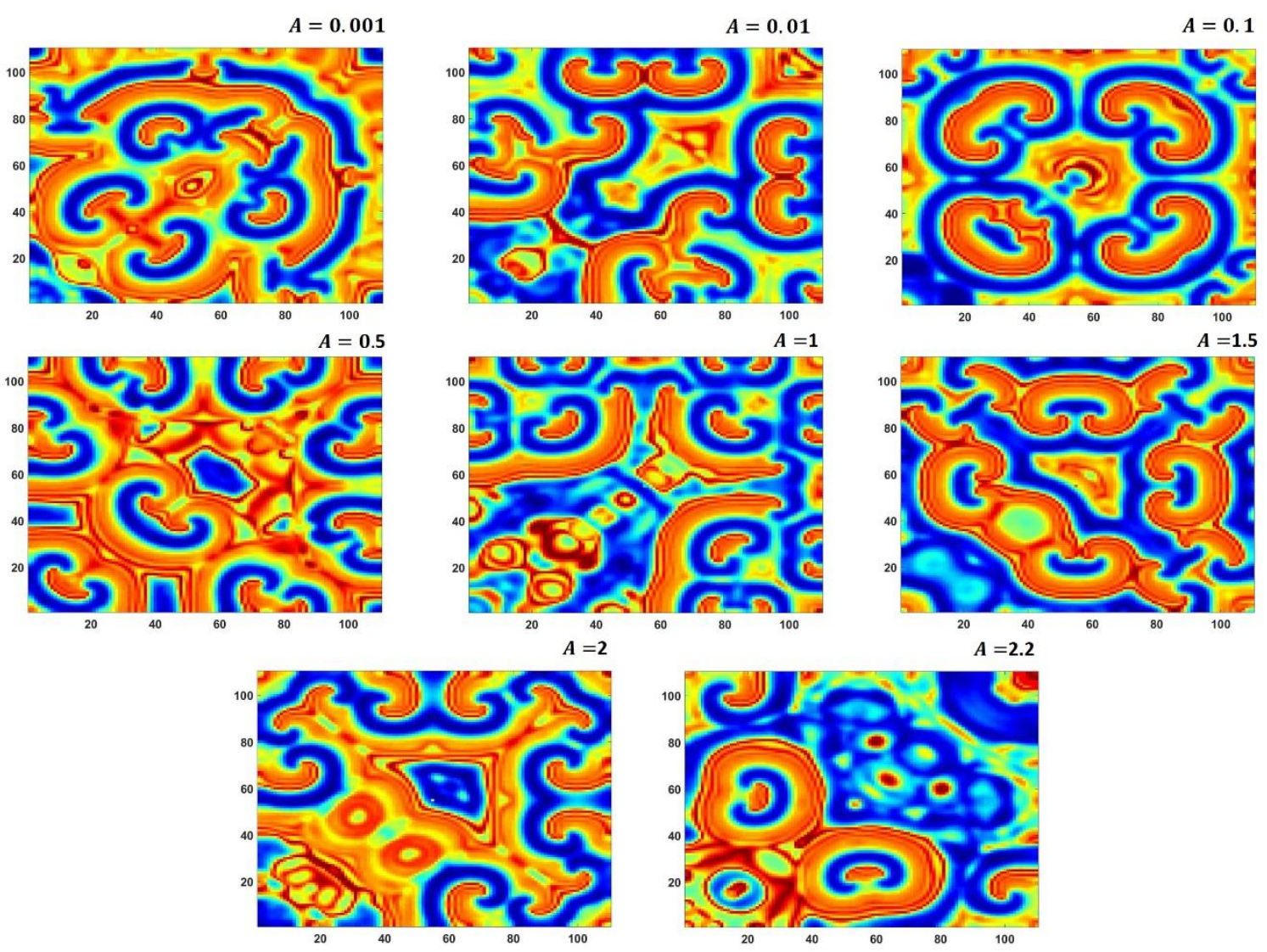

Fig.13: Spatiotemporal snap shots of the membrane potential for Type-1 non-autonomous (Case-B) EMLN network for various values of the stimuli amplitude $A$ with $\omega=0.1$ and $D=1$. The required parameters for simulation are $C=1, V_{C a}=1, V_{L}=-0.5, g_{C a}=$ $1.2, g_{K}=2, g_{L}=0.5, V_{0}=0.2, V_{1}=-0.01, V_{2}=0.15, V_{3}=0.1, V_{4}=0.05, \sigma=3, \varepsilon=$ $0.1, k_{0}=0.1, k_{1}=0.2, k_{2}=1, \alpha=0.1, \beta=0.1, E_{1}=1, \omega_{1}=1$

\subsubsection{Effect of the stimuli frequency $\omega$}

Comparing the effect of stimuli frequency on the externally excited type-1 EMLN network largely varies when compared to the non-excited network shown in Fig.10. As the system is already excited, the stimuli doesn't have any effect on the wave propogation and to show this we have displayed the spatiotemporal snapshots as in Fig.14. As seen in the figure the stimuli frequency doesnot the generate spiral waves even after the value of $\omega$ is increased over 50, 
which is 10 times higher than the value of $\omega=5$ at which the spiral waves diminish for the autonomous case as shown in Fig.10.
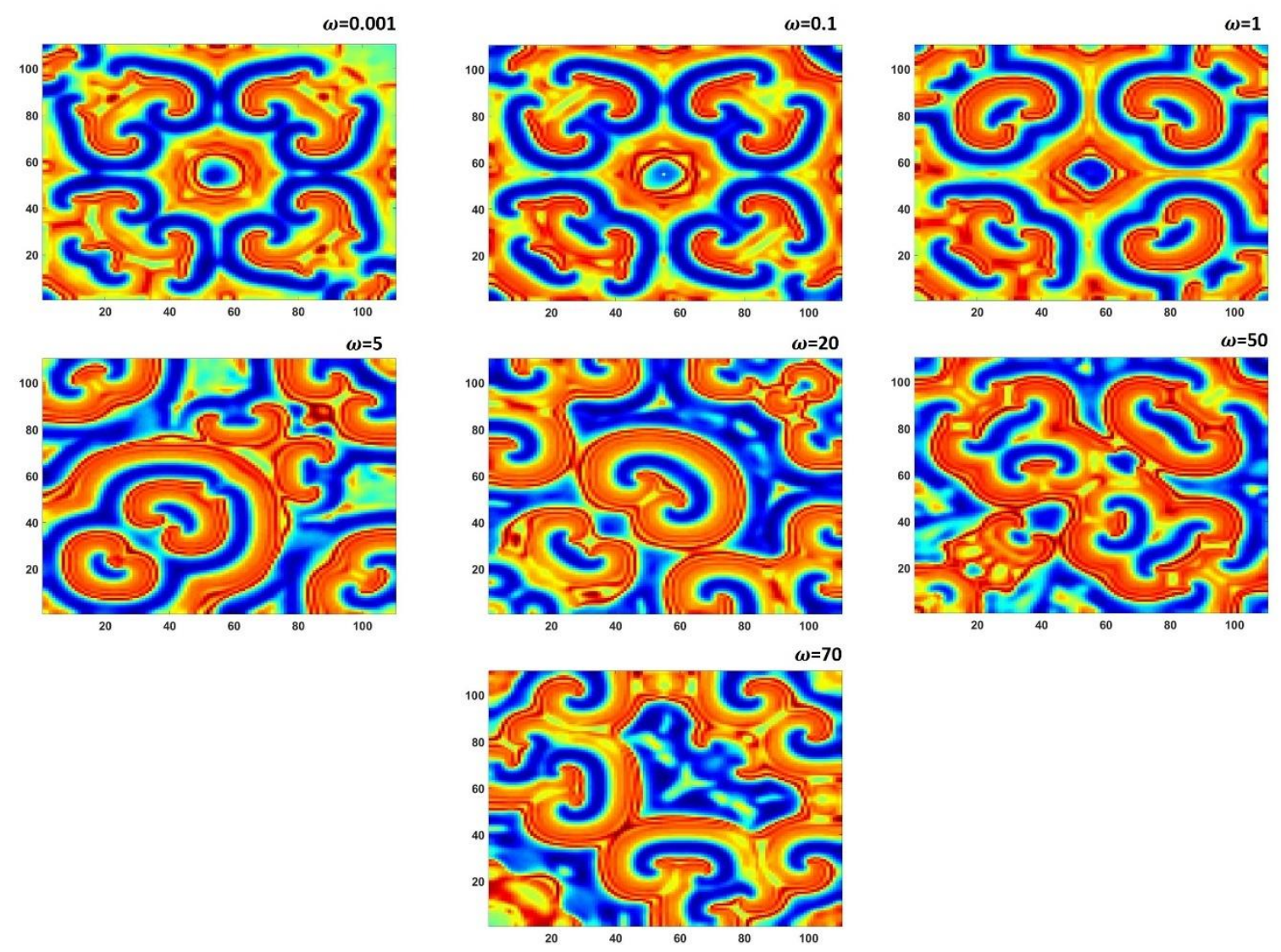

Fig.14: Spatiotemporal snap shots of the membrane potential for Type-1 non-autonomous (Case-B) EMLN network for various values of the stimuli frequency $\omega$ with $A=0.1$ and $D=1$. The required parameters for simulation are $C=1, V_{C a}=1, V_{L}=-0.5, g_{C a}=$ $1.2, g_{K}=2, g_{L}=0.5, V_{0}=0.2, V_{1}=-0.01, V_{2}=0.15, V_{3}=0.1, V_{4}=0.05, \sigma=3, \varepsilon=$ $0.1, k_{0}=0.1, k_{1}=0.2, k_{2}=1, \alpha=0.1, \beta=0.1, E_{1}=1, \omega_{1}=1$

\subsubsection{Effect of the coupling strength D}

Coupling strengths between the neighboring neurons play a vital role in the wave propagation phenomenon. It was earlier proved in the literature that the larger is the coupling the greater is the possibility of spiral waves. Fig. 15 shows the snapshots of the network for various coupling strengths, it can be seen that for coupling strength greater than $50 \%$, we see the emergence of spiral waves. 

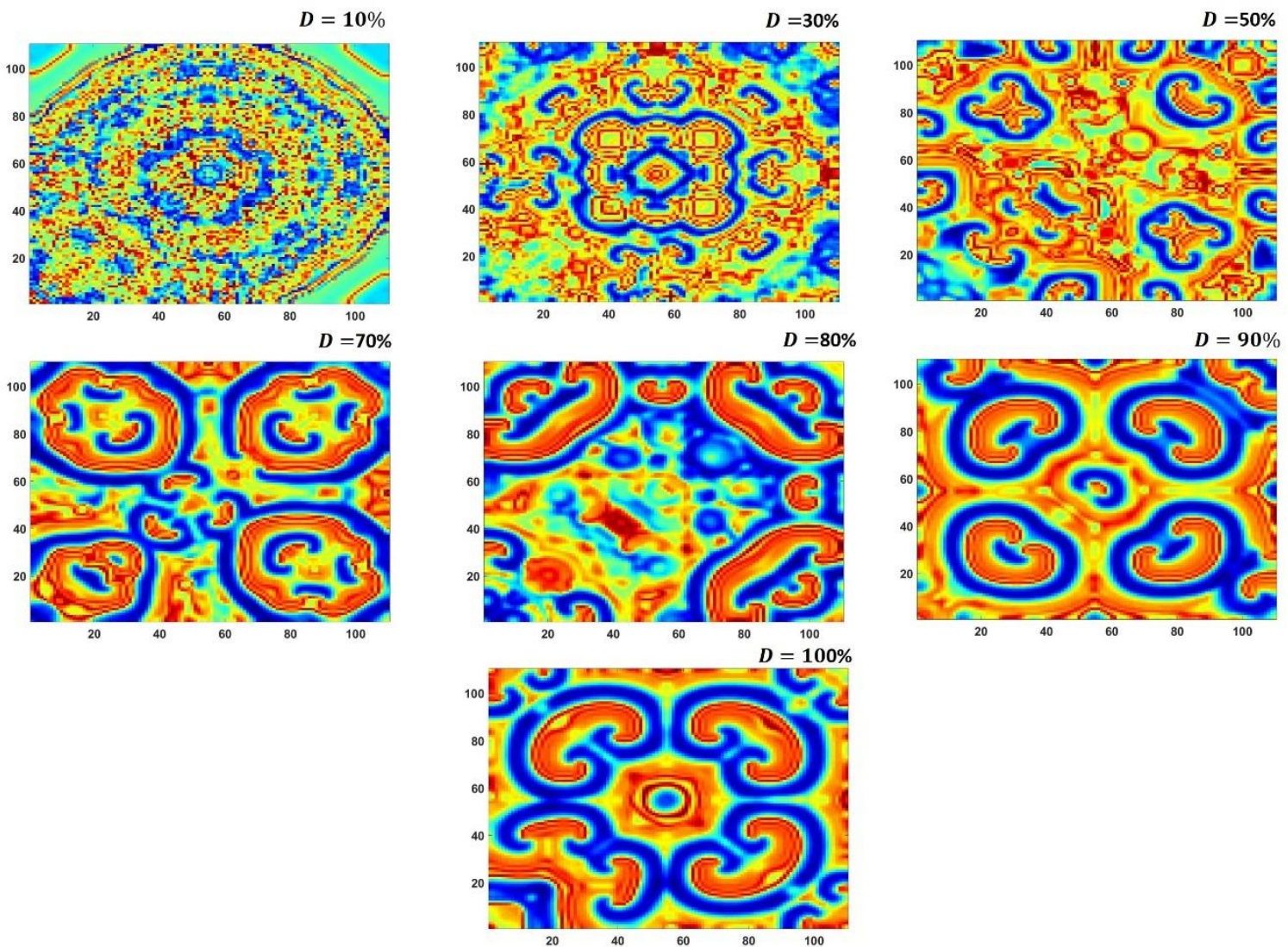

Fig.15: Spatiotemporal snap shots of the membrane potential for Type-1 non-autonomous (Case-B) EMLN network for various values of the coupling strength $D$ with $\omega=0.1$ and $A=0.1$. The required parameters for simulation are $C=1, V_{C a}=1, V_{L}=-0.5, g_{C a}=$ $1.2, g_{K}=2, g_{L}=0.5, V_{0}=0.2, V_{1}=-0.01, V_{2}=0.15, V_{3}=0.1, V_{4}=0.05, \sigma=3, \varepsilon=$ $0.1, k_{0}=0.1, k_{1}=0.2, k_{2}=1, \alpha=0.1, \beta=0.1, E_{1}=1, \omega_{1}=1$

\subsubsection{Effect of the Nernst potential $V_{K}$}

Our final investigation is concerned with the bifurcation parameter $V_{K}$ of the EMLN system. By fixing the external stimuli amplitude and frequency at 0,1 respectively and maintaining $100 \%$ coupling between the neurons, we study the spatiotemporal behaviors of the network for various values of $V_{K}$, As seen from Fig.16, the spiral waves exist only for a small range of $-450 \leq V_{K} \leq-350$ which are equally supported by the forward continuation bifurcation plot shown in Fig.7a as blue dots. This evidence of nonexistence of spiral waves for $V_{K} \leq-450$ clearly shows that the generation of spiral waves depends mainly on the chaotic behavior of the system in the network. 

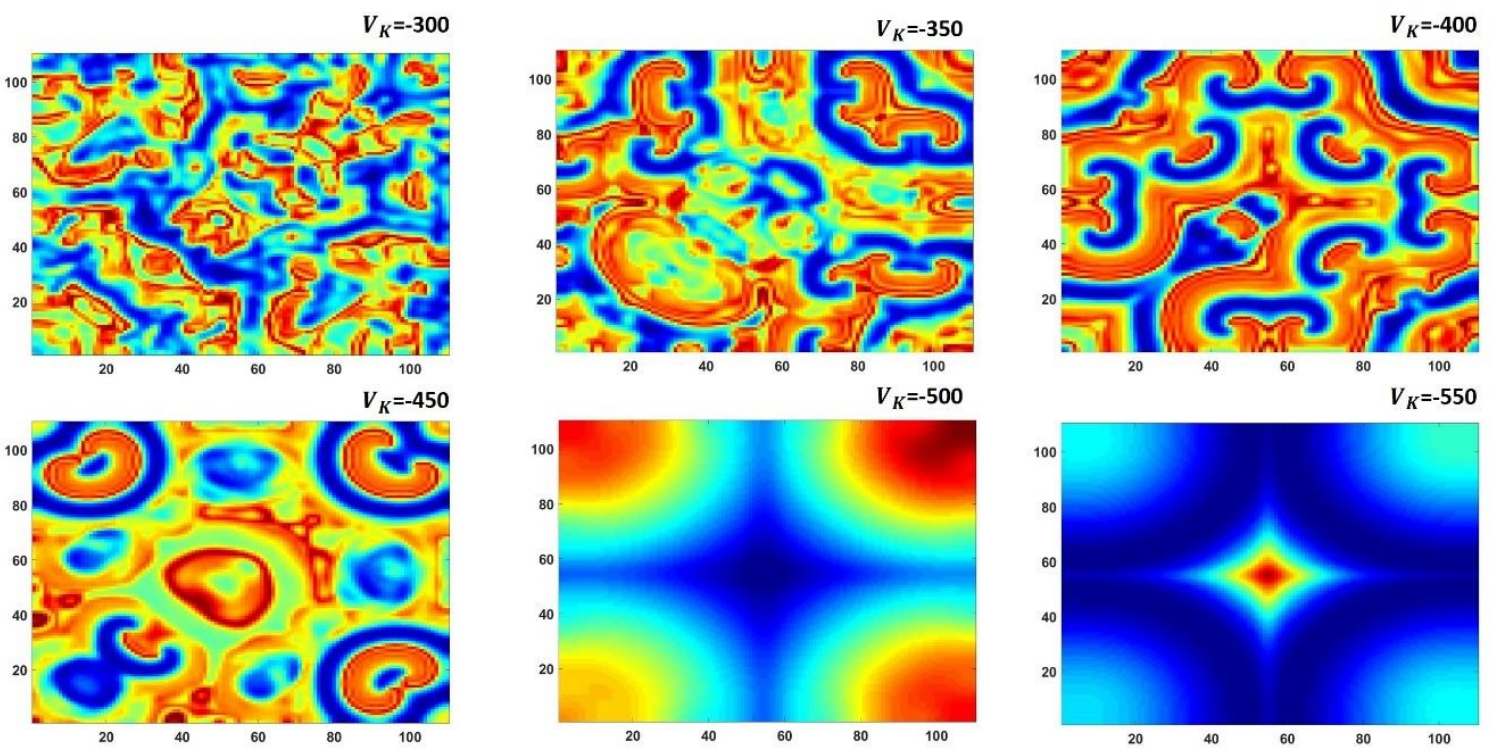

Fig.16: Spatiotemporal snap shots of the membrane potential for Type-1 non-autonomous (Case-B) EMLN network for various values of the Nernst potential $V_{K}$ with $A=0.1, \omega=0.1$ and $D=1$. The required parameters for simulation are $C=1, V_{C a}=1, V_{L}=-0.5, g_{C a}=$ 1.2, $g_{K}=2, g_{L}=0.5, V_{0}=0.2, V_{1}=-0.01, V_{2}=0.15, V_{3}=0.1, V_{4}=0.05, \sigma=3, \varepsilon=$ $0.1, k_{0}=0.1, k_{1}=0.2, k_{2}=1, \alpha=0.1, \beta=0.1, E_{1}=1, \omega_{1}=1$

\subsection{Wave propagation in a type-2 network:}

For this analysis we consider that the neurons in the network are in a chaotic state for the first 2000-time units by keeping the Nernst potential of potassium $\left(V_{K}\right)$ at -410 and for the remaining 3000 -time units $(2000 \leq t \leq 5000)$ we consider that the neurons are in either periodic or chaotic states by choosing different values of $V_{K}$. The investigation is conducted on both autonomous and non-autonomous type-2 EMLN network shown in (12). The amplitude and frequency of the stimuli is taken as 0.1 each and coupling is $100 \%$. In Fig. 17 we have shown the snapshots of the wave propagation in the network (12) taking different values of $V_{K}$ for $2000 \leq t \leq 5000$. Comparing Fig.12 with Fig.17, we see that in Fig.12, the spiral waves die for values of $V_{K}<-550$ whereas in Fig. 17 we see that the spiral waves exist for even much lower potential of $V_{K}=-800$. It is to be noted that when the systems exhibit time dependent multistability, spiral waves are seen even in non-chaotic regions. Such inferences were never reported earlier. 

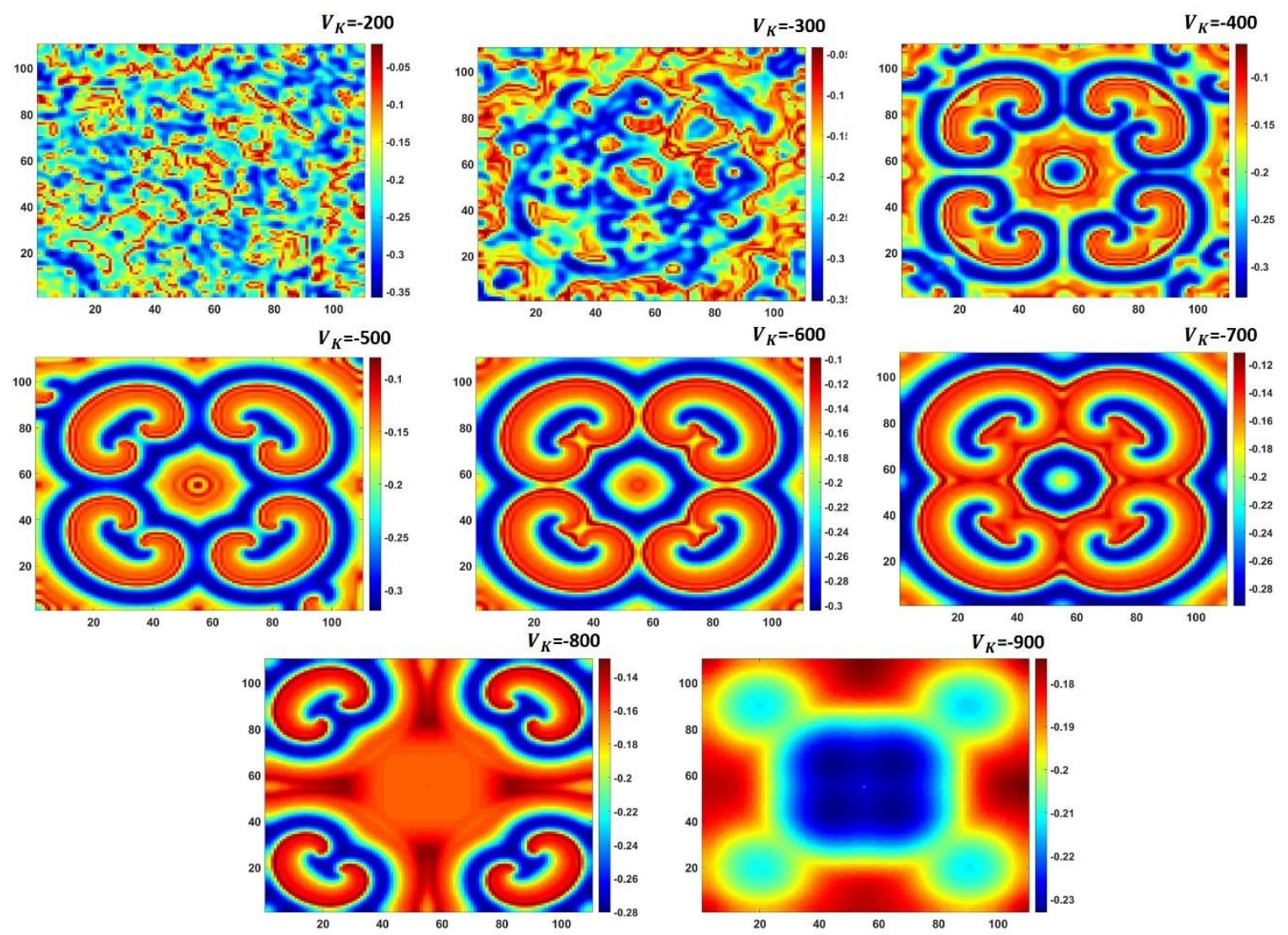

Fig.17: Spatiotemporal snap shots of the membrane potential for Type-2 autonomous (CaseA) EMLN network for various values of the Nernst potential $V_{K}$ with $A=0.1, \omega=0.1$ and $D=1$. The required parameters for simulation are $C=1, V_{C a}=1, V_{L}=-0.5, g_{C a}=$ 1.2, $g_{K}=2, g_{L}=0.5, V_{0}=0.2, V_{1}=-0.01, V_{2}=0.15, V_{3}=0.1, V_{4}=0.05, \sigma=3, \varepsilon=$ $0.1, k_{0}=0.1, k_{1}=0.2, k_{2}=1, \alpha=0.1, \beta=0.1, E_{1}=1, \omega_{1}=1$

Similarly, we investigate the behaviors of the type-2 EMLN network in the presence of external excitation. Fig. 18 shows the snapshots of the spatiotemporal behavior. We have already shown that in the presence of external excitation the spiral waves are seen only for a smaller bandwidth $-450 \leq V_{K} \leq-350$, where as Fig. 18 shows that when considering the network as chaotic for the first 2000-time units, the spiral wave region extends to as low as $V_{K}=-800$. 

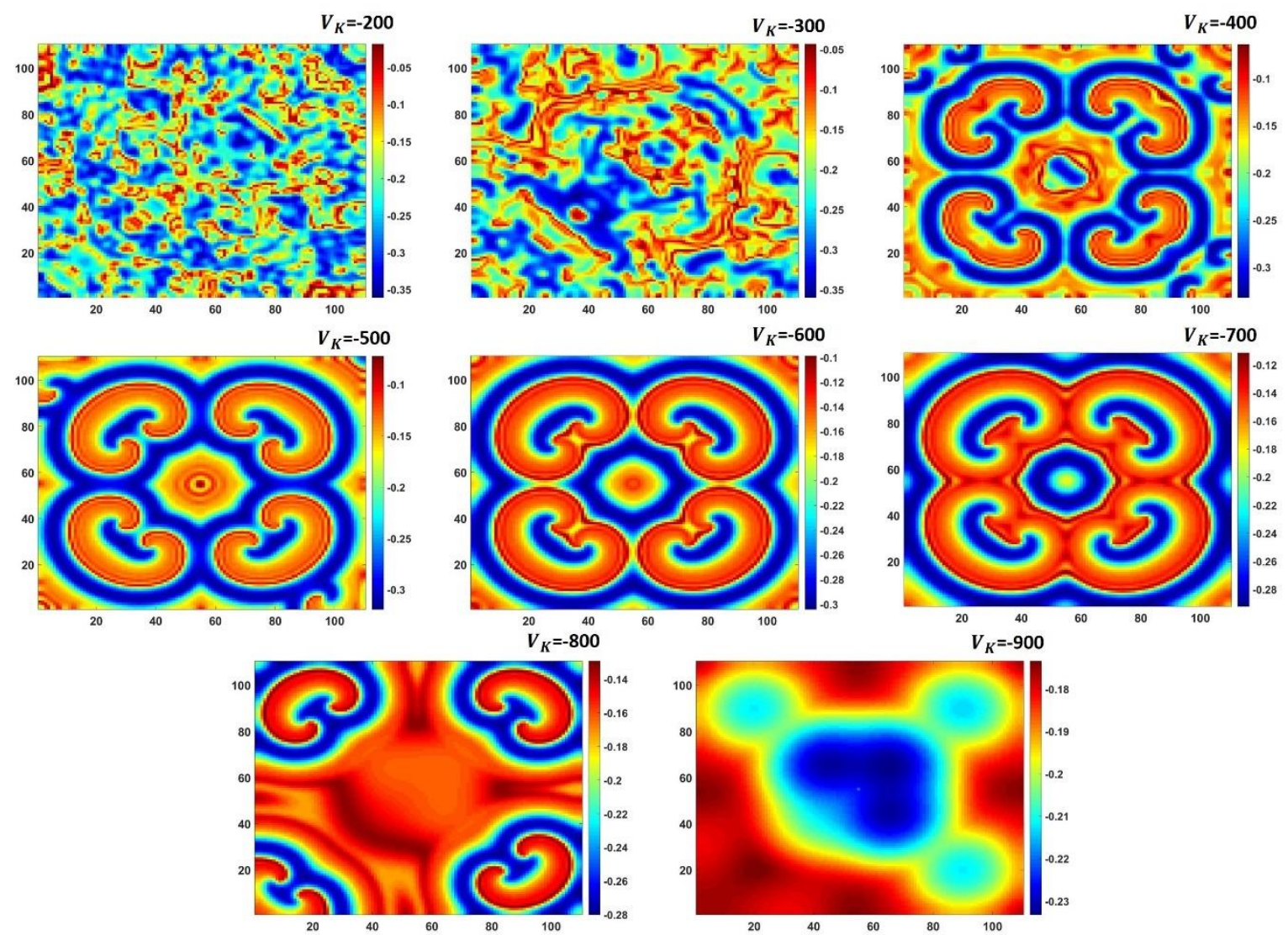

Fig.18: Spatiotemporal snap shots of the membrane potential for Type-2 non-autonomous (Case-B) EMLN network for various values of the Nernst potential $V_{K}$ with $A=0.1, \omega=0.1$ and $D=1$. The required parameters for simulation are $C=1, V_{C a}=1, V_{L}=-0.5, g_{C a}=$

$$
\begin{gathered}
1.2, g_{K}=2, g_{L}=0.5, V_{0}=0.2, V_{1}=-0.01, V_{2}=0.15, V_{3}=0.1, V_{4}=0.05, \sigma=3, \varepsilon= \\
0.1, k_{0}=0.1, k_{1}=0.2, k_{2}=1, \alpha=0.1, \beta=0.1, E_{1}=1, \omega_{1}=1
\end{gathered}
$$

\section{Conclusion}

In this study we used computer simulations to investigate a new extended version of the MorrisLecar neuron with the electromagnetic flux coupling and external excitation. The new model shows more richer dynamical properties like multistability and coexisting attractors in the absence and presence of external excitation. It has been very well discussed in the literature that the Morris-Lecar neuron model very much replicates the physical behavior of real neurons. Literature have shown that the emergence of spiral waves in cardiac tissues can lead to arrhythmias and similarly cortical spiral waves are the reasons for the epileptic seizures. Also breaking up of spiral seeds into multiple spiral pools results in sudden non-synchronous behavior of heart muscle fibers which prevents the heart from pumping the purified blood. This is commonly known as fibrillation and results in higher mortalities. Normally spiral patterns are formed by the reentrant waves into an excitable media. Hence, we were interested in investigating the formation of spiral waves in a lattice neuron network whose local dynamics is governed by the EMLN model. Studying the nature of spiral waves and finding ways to eliminate these waves are important and to do this we discuss the effect of the stimuli force on the spiral wave formations in the presence and absence of external excitations. We have shown 
that in the absence of external excitation, the spiral waves are easily controlled and eliminated by properly selecting the stimuli frequency as in our case for $\omega \geq 5$ the spiral waves are dissipated by the stimuli force. But this was not in the case when we consider external excitation as even for large stimuli frequency of $\omega=70$, the spiral waves are seen in the network. But when we change the stimuli amplitude, we see the spiral wave dissipation but when $A=2.3$ the entire network goes unbounded and thus we cannot conclude the effect of the stimuli force on the network. It was discussed in literatures that low frequencies cannot break the reorganizing rhythm of the spiral seeds, while the higher stimuli frequencies can break spiral rhythm. When external excitation is considered even the higher stimuli frequencies cannot break the spiral rhythm. As we have shown in the dynamical properties, the Nernst potential of the potassium ions are considered as the control parameter and we constructed a time changing network whose dynamics changes to periodic to chaotic or vice-versa. To achieve this, we have change the value of $V_{K}$ and have investigated the spatiotemporal kinetics of the network. We could show that irrespective of the type of external excitation the spiral waves are seen in the network to values of $V_{K}=-800$ which cannot be seen in the type-1 network. Thus, in special cases when the neurons are not in previous states, the spiral wave exists for values which have not been much reported earlier.

Conflict of Interest: The authors declare that they have no conflict of interest.

Funding: There is no funding received for this study.

\section{References}

[1] FitzHugh R.: Mathematical models of threshold phenomena in the nerve membrane. Bull. Math. Biophysics, 17, 257-278 (1955)

[2] FitzHugh R.: Impulses and physiological states in theoretical models of nerve membrane. Biophysical J, 1, 445-466 (1961)

[3] FitzHugh R.: Mathematical models of excitation and propagation in nerve. Chapter 1 (pp. 1-85 in H.P. Schwan, ed. Biological Engineering, McGraw-Hill Book Co., N.Y.). (1969)

[4] Nagumo J., Arimoto S., and Yoshizawa S.: An active pulse transmission line simulating nerve axon. Proc IRE. 50, 2061-2070 (1962)

[5] Hodgkin, A., and A. Huxley.: Currents carried by sodium and potassium ions through the membrane of the giant axon of Loligo. J Physiol. 116(4), 449-472 (1952)

[6] Morris, C, Lecar.H.: Voltage Oscillations in the Barnacle Giant Muscle Fiber. Biophysical Journal 35(1), 193-213 (1981)

[7] Pei-Li Chen.: Some aspects of the Morris-Lecar model and the myelinated axon models with Morris-Lecar dynamics. Mathl. Comput. Modelling 17(8), 85-97 (1993)

[8] Tsumoto, K., Kitajima, H., Yoshinaga, T., Aihara, K, Kawakami, H.: Bifurcations in Morris-Lecar neuron model. Neurocomputing 69, 293-316 (2006)

[9] Wang, H. X., Lu, Q. S. \& Wang, Q. Y.: Generation of firing rhythm patterns and synchronization in the Morris-Lecar neuron model. Int. J. Nonlin. Sci. Numer. Simulat. 6, 7-12 (2005) 
[10] Wang, H. X., Lu, Q. S. \& Wang, Q. Y.: Bursting and synchronization transition in the coupled modified M-L neurons. Commun. Nonlin. Sci. Numer. Simulat. 13, 1668-1675 (2008)

[11] Xinlin Song, Hengtong Wang, Yong Chen.: Autapse-induced firing patterns transitions in the Morris-Lecar neuron model. Nonlinear Dyn, 2019. DOI:10.1007/s11071-01904925-7

[12] Farjami. S, Kirk. V, Osinga. H. M.: Interactions between a locally separating stable manifold and a bursting periodic orbit. Eur. Phys. J. Special Topics, 227, 603-614 (2018)

[13] Vetriveeran Rajamani, Maheshwar PD. Sah, Zubaer Ibna Mannan, Hyongsuk Kim, Leon Chua.: Third-Order Memristive Morris-Lecar Model of Barnacle Muscle Fiber. International Journal of Bifurcation and Chaos, 27(4) 1730015 (2017)

[14] Congmin Liu, Xuanliang Liu, Shenquan Liu.: Bifurcation analysis of a Morris-Lecar neuron model", Biol Cybern, 108, 75-84 (2014)

[15] Prescott SA, De Koninck Y, Sejnowski TJ.: Biophysical basis for three distinct dynamical mechanisms of action potential initiation PLOS Comput Biol 4, 1-18 (2008)

[16] Bocheng Bao, Qinfeng Yang, Lei Zhu, Han Bao, Quan Xu, Yajuan Yu, Mo Chen.: Chaotic Bursting Dynamics and Coexisting Multistable Firing Patterns in 3D Autonomous Morris-Lecar Model and Microcontroller-Based Validations. International Journal of Bifurcation and Chaos, 29(10), 1950134-1950152, (2019)

[17] Alexandre Wagemakers, miguel A. F. Sanjuan, Jose M. Casado, Kazuyuki Aihara.: Building Electronic Bursters With The Morris-Lecar Neuron Model. International Journal of Bifurcation and Chaos, 16(12), 3617-3630 (2006)

[18] Jiang Wang, Meili Lu, Huiyan Li.: Synchronization of coupled equations of MorrisLecar model. Communications in Nonlinear Science and Numerical Simulation 13, 1169-1179 (2008)

[19] Y. Kuramoto and D. Battogtokh.: Coexistence of Coherence and Incoherence in Nonlocally Coupled Phase Oscillators. Nonlinear Phenomena in Complex Systems 5(4), 380-385 (2002)

[20] S. Shima and Y. Kuramoto.: Rotating spiral waves with phase-randomized core in nonlocally coupled oscillators. Physical Review E 69, 036213 (2004)

[21] Y. Kuramoto and S. Shima.: Rotating Spirals without Phase Singularity in ReactionDiffusion Systems. Progress of Theoretical Physics Supplement, 150, 115 (2003)

[22] Y. Kuramoto, S. Shima, D. Battogtokh, and Y. Shiogai.: Mean-Field Theory Revives in Self-Oscillatory Fields with Non-Local Coupling. Progress of Theoretical Physics Supplement 161, 127 (2006)

[23] Erik A. Martens, Carlo R. Laing, Steven H. Strogatz.: Solvable Model of Spiral Wave Chimeras. Physical Review Letters (2010)

[24] Samie F H, Mandapati R, Gray R A, Watanabe Y, Zuur C.: A Mechanism of Transition From Ventricular Fibrillation to Tachycardia. Circ Res 86, 684-691 (2000)

[25] Samie F H, Jalife J.: Mechanisms underlying ventricular tachycardia and its transition to ventricular fibrillation in the structurally normal heart. Cardiovasc Res 50, 242-250, (2001)

[26] Yuan G Y, Wang G R, Chen S G.: Control of spiral waves and spatiotemporal chaos by periodic perturbation near the boundary. Europhys Lett 72, 908 (2005) 
[27] Fenton F H, Cherry E M, Hastings H M, Evans S J.: Multiple mechanisms of spiral wave breakup. Chaos 12, 852-892 (2002)

[28] Aranson I, Kessler D, Mitkov I.: Drift of spiral wave in excitable media. Physica D 85, $142-155$ (1995)

[29] Zykov V S.: Kinematics of rigidly rotating spiral waves. Physica D 238, 931-940 (2009)

[30] Heather A, Brooks, Paul C. Bressloff.: Quasicycles in the stochastic hybrid Morris-Lecar neural model. Physical Review E 92, 012704 (2015)

[31] Hou Z H, Xin H W.: Noise-sustained spiral waves: effect of spatial and temporal memory. Phys Rev Lett 89, 280601 (2002)

[32] Gu H G, Jia B, Li Y Y,Chen G R.: White noise-induced spiral waves and multiple spatial coherence resonances in a neuronal network with type I excitability. Physica A (2013)

[33] Ali Calim, Philipp Hövel, Mahmut Ozer, Muhammet Uzuntarla.: Chimera states in networks of type-I Morris-Lecar neurons. Physical Review E 98, 062217 (2018)

[34] Xinyi Wu, Jun Ma.: The Formation Mechanism of Defects, Spiral Wave in the Network of Neurons. Plos One, 8(1) (2013)

[35] Izhikevich, E. M.: Neural excitability, spiking and bursting. Int. J. Bifurcation and Chaos 10, 1171-1266 (2000)

[36] Lv M , Ma J .: Multiple modes of electrical activities in a new neuron model under electromagnetic radiation. Neurocomput 2016, 205(3), 75-81 (2016)

[37] Karthikeyan Rajagopal, Fahimeh Nazarimehr, Anitha Karthikeyan, Ahmed Alsaedi, Tasawar Hayat, Viet-Thanh Pham.: Dynamics of a neuron exposed to integer order and fractional order discontinuous external magnetic flux. Frontiers of Information Technology \& Electronic Engineering, 20, 584-590 (2019)

[38] Fatemeh Parastesh, Karthikeyan Rajagopal, Fawaz E. Alsaadi, Tasawar Hayat, V.-T. Pham, Iqtadar Hussain.: Birth and Death of spiral waves in a network of Hindmarsh-Rose neurons with exponential magnetic flux and excitable media. Applied Mathematics and Computation, Applied Mathematics and Computation, 354, 377-384 (2019)

[39] Karthikeyan Rajagopal, Fatemeh Parastesh, Hamed Azarnoush, Boshra Hatef, Sajad Jafari and Vesna Berec.: Spiral waves in externally excited neuronal network: solvable model with a monotonically differentiable magnetic flux. Chaos: An Interdisciplinary Journal of Nonlinear Science, 29(4), 043109 (2019)

[40] Rajagopal, K, Khalaf, A.J.M., Parastesh, F., et al.: Dynamical behavior and network analysis of an extended Hindmarsh-Rose neuron model, Nonlinear Dyn 98, 477-487 (2019)

[41] Jun Ma, Ya Wang, Chunni Wang, Ying Xu, Guodong Ren.: Mode selection in electrical activities of myocardial cell exposed to electromagnetic radiation. Chaos, Solitons and Fractals 99, 219-225 (2017)

[42] A. Wolf, J. B. Swift, H. L. Swinney, J. A. Vastano.: Determining Lyapunov exponents from a time series. Physica D: Nonlinear Phenomena, 16, 285-317 (1985)

[43] Wu, F., Ma, J, Zhang, G.: Energy estimation and coupling synchronization between biophysical neurons. Science China Technological Sciences, 63, 625-636 (2020)

[44] Ma, J., Yang, Z., Yang, L., Tang, J.: A physical view of computational neurodynamics. Journal of Zhejiang University-SCIENCE A, 20(9), 639-659 (2019) 